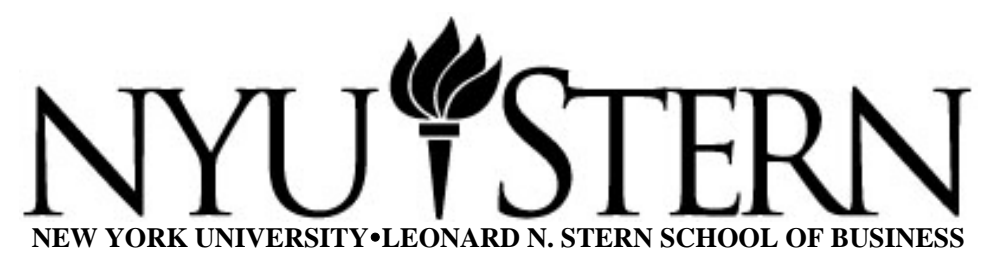

Department of Finance

Working Paper Series

FIN-03-008

\title{
Market Size and Investment Performance of Defaulted Bonds and Bank Loans: 1987-2002
}

\author{
Edward I. Altman and Shubin Jha
}

February 2003

The 2003 NYU Stern Department of Finance Working Paper Series is generously sponsored by

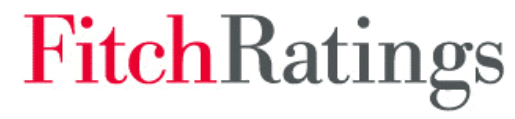




\title{
Report on
}

\section{Market Size and Investment Performance of Defaulted Bonds and Bank Loans: 1987-2002}

\author{
by \\ Edward I. Altman \\ with \\ Shubin Jha
}

\section{February 2003}

Dr. Altman is the Max L. Heine Professor of Finance and Vice Director of the NYU Salomon Center, Leonard N. Stern School of Business. Mr. Jha is a Research Associate at the NYU Salomon Center. The assistance of Gaurav Bana, Deepak-Iyer Ramamurthy, Amit Arora and Lourdes Tanglao of the NYU Salomon Center and the many securities firms and distressed securities investors who provided us with price quotations and other data is appreciated. Special thanks to Gabriella Petrucci, Sau Man Kam and Wilson Miranda of Salomon Smith Barney for their data assistance. 


\title{
Market Size and Investment Performance of Defaulted Bonds \& Bank Loans: \\ 1987-2002
}

\begin{abstract}
- The defaulted and distressed, public and private debt markets in the United States increased enormously to a record $\$ 942$ billion (face value) at the end of 2002. The market value of this increasingly attractive alternative investment segment was approximately $\$ 512$ billion.

- Defaulted securities performed below average in 2002; absolute returns, as measured by our various defaulted debt indexes, were $-6.0 \%$ on bonds, $+3.0 \%$ on bank loans, and $-0.5 \%$ on the combined defaulted public bonds and private bank loans index.

- The Altman-NYU Salomon Center Index of Defaulted Bonds grew to a face value of $\$ 61.5$ billion. The market-to-face value ratio of the Bond Index fell to 0.17 from 0.21 one year ago. The face value of our Defaulted Bank Loan Index was $\$ 37.7$ billion and the market-to-face value ratio dropped to a record low level of 0.46 by the end of 2002 .

- The recovery rate on defaulted bonds (price just after default) was very low at 25 cents on the dollar; likewise, the weighted average bank loan recovery rate in 2002 dropped to 52 cents on the dollar. With new defaulted bonds rising in 2002 to a record $\$ 96.9$ billion (default rate of $\mathbf{1 2 . 8 \%}$ ) and the default outlook for 2003 high, but lower than for 2002, investment opportunities should abound in the distressed debt market.

- Indications are that distressed investors (both old and new entities) are successfully raising funds because investor expectations are buoyant.
\end{abstract}




\section{Introduction}

This report on the size and performance of defaulted bonds and bank loans presents our annual update and analysis. For in-depth discussions of the supply and demand elements of defaulted and distressed securities, as well as their performance and other attributes, see our annuals reports (1993-2001), Altman (1991, 1993); Branch and Ray (1992); Altman \& Eberhart (1994); Ward \& Griepentrog (1993); Gilson (1995); Hotchkiss and Mooradian (1998); Reilly, Wright and Altman (1998); and Eberhart, Altman and Aggarwal (1999). Defaulted bonds and bank loans performed somewhat poorly during 2002, reversing the relatively good performance of the preceding year, but more in line with several of the past recent years. This "asset class" has attracted an increasing amount of new capital, however, as the supply of distressed and defaulted debt securities continued its substantial growth over the past four years. Indeed, the estimated supply of

defaulted public and private, distressed and defaulted, debt reached an enormous total of $\$ 942$ billion by year-end 2002 . 


\section{Size of the Defaulted and Distressed Debt Market}

The size of the defaulted and distressed debt market again grew in 2002 to its largest size since we have been indexing this asset class, topping even the extraordinary levels of 2001. Figure 1 exhibits the significant amount of newly defaulted bonds during 2002 ( $\$ 96.9$ billion) which grew by more than $50 \%$ above 2001 levels ( $\$ 63.6$ billion) and more than tripled the total of 2000. At the end of 2002, the distressed proportion of the total high yield market (including defaulted securities) was similar to last year's proportion (21\%) and the defaulted proportion grew to 19\% (Figure 2). The supply of distressed high yield bonds (defined as yield to maturity greater than 1,000 basis points over ten-year Treasuries) increased in 2002 to \$205 billion from \$160 billion in 2001. The total face value amount of defaulted and distressed public bonds outstanding grew from 2001's previous record level of \$257 billion to \$393 billion (Figure 3).

Assuming an average private to public debt ratio of 1.40, down from 1.65 in 2001 and 2.0 in 1999, the level of public and private defaulted and distressed debt is estimated to have reached a face value of $\$ 942$ billion, easily topping 2001's record amount of $\$ 680$ billion. We have dropped our market value benchmark to 0.20 (from 0.25 ) and 0.50 of face value for public defaulted and distressed bonds, respectively. The resulting estimated market value for distressed and defaulted debt increased sharply to just over $\$ 512$ billion. The trend of these amounts from 1990-2002 is shown in Figure 4 (certain years are not indicated since we did not analyze the market in all years).

We expect a 7.5\%-8.0\% default rate on high yield bonds in 2003 (see our partner Annual Report "Defaults and Returns on High Yield Bonds," NYU Salomon Center Report, February 2003). This will add to our base supply of defaulted securities. And, if 
the default rate has, in fact, peaked in the third quarter of 2002, as we believe, this will probably be good news for distressed investors as the supply of new distressed debt should subside somewhat relative to a growing demand. The latter is roughly estimated to be in the $\$ 50-\$ 60$ billion range as of year-end 2002 .

\section{Measuring and Monitoring Performance of Defaulted Debt}

\section{Defaulted Bond Index}

The Altman-NYU Salomon Center Defaulted Bond Index (A-NYU Index) was developed in 1990 for the purpose of measuring and monitoring the performance of defaulted debt securities. ${ }^{1}$ The sample period of our Index begins in January 1987 and, as of December 31, 2002, includes 166 issues from 113 firms (Figure 5). ${ }^{2}$ The Index's market value was $\$ 10.4$ billion and its face value was $\$ 61.6$ billion. The size of our Index, as measured by the face value of public defaulted bonds, is more than triple the face value of the Index during the early 1990s; yet the market value of our Index is actually slightly lower than its highest measure, previously observed in 1992 . Figure 5 exhibits various measures of our Index's size since its December 1986 inception. The variability in the number of issues, with a low of 30 in 1986 and a high of 231 in 1992 , continues to be notable. Our expectation that the huge new issue supply of noninvestment grade debt in the years 1996-1999 would result in a continued increase of default amounts during subsequent years was again realized in 2002 .

\footnotetext{
${ }^{1}$ This index, originally developed in Altman's Foothill Report (1990) is maintained and published on a monthly basis at the NYU Salomon Center of the Leonard N. Stern School of Business. It is available, along with data and reports on high yield debt default rates and performance, from the Center (212-9980701 or 212-998-0709).

${ }^{2}$ The number of issues in 2001 was actually somewhat higher but many were not trading regularly and were relatively small. We have trimmed the list of these illiquid issues. Note that the number of firms in 2002 (113) were greater, however, than last year's total (86).
} 


\section{Defaulted Bank Loan Index}

Managers of distressed securities are more commonly investing in both distressed bonds and the private debt (particularly bank debt) of defaulting companies. The observed increasing investment in defaulted private debt has been coincident with the bank loan market's increasing size and liquidity as market makers have devoted considerable resources to bank debt trading. In 2002, for the first time, there was more trading in distressed bank debt (loans trading at or below 90 cents on the dollar) than in non-distressed loans (from Loan Pricing Corporation data). We have responded by calculating an Index of Defaulted Bank Debt Facilities, as well as a Combined Index of bonds and bank loans.

The Altman-NYU Salomon Center Index of Defaulted Bank Loans, like the defaulted bond index, is a market-weighted, monthly total return index comprised of U.S. companies. The Index contained 17 facilities at its inception in December 1995 and has grown to reach 141 facilities from 56 borrowers as of December 31, 2001 and 51 borrowers at the end of 2002 (Figure 6).

\section{Market/Face Value Ratios}

We consider the ratio of the aggregate market value to face value of the component securities that comprise our indexes to be an important measure of the defaulted debt markets' current relative health and potential future returns. This ratio has ranged, at year-end for defaulted bonds, from a maximum level of 0.74 in 1987 to a minimum level of 0.15 in 2000 (Figure 5). While the market/face value ratio has varied within a fairly narrow range of 0.30 to 0.55 during a majority of years in our 17 -year sample period (1986-2002), abnormal returns for the Index have resulted in a numbers of 
market/face value ratio observations well outside of this range. Indeed, the ratio has been 0.25 or below in each of the last five years and ended 2002 at 0.17 , down from 0.21 one year earlier. The trend in the market/face value ratios of both defaulted bonds and bank loans can be seen clearly in Figure 7. Note that the Defaulted Bank Loan Index dropped to its all time low ratio in 2002 to 0.46 .

One can conclude that if there is movement in the future in the average price level of both defaulted bonds and bank loans that resemble a regression-to-the-mean, then both indexes should rise. In the interest of full disclosure, we have noted this relationship in the past and have not observed the anticipated significant increase in prices and returns. We still believe that both indexes are at usually low levels, which bodes well for returns in 2003.

\section{Performance Measurement}

The A-NYU Index includes the securities of firms at various stages of reorganization either in bankruptcy or restructuring. We calculate the returns for the Index using data compiled from just after default to the point when the bankrupt firm emerges from Chapter 11, is liquidated, or until the default is "cured" or resolved through an exchange. The securities of distressed restructured companies are also included in the Index. The Index included bond issues of all seniorities, from senior-secured to juniorunsecured debt. A study by Altman and Eberhart (1994), updated by Standard \& Poor's (Brand and Behar, 1998), measures the performance of defaulted debt from the time of original issuance through default and then to emergence from bankruptcy. These studies conclude that the seniority of the issue is an extremely important determinant of the performance of defaulted securities over specific periods, whether from issuance to 
emergence or from default to emergence. The A-NYU Index does not include convertible or international company issues, nor does it include distressed, but not defaulted, securities.

\section{Performance of Defaulted Bonds}

The Altman-NYU Salomon Center Index of Defaulted Bonds reversed its excellent performance in 2001 , falling by $5.98 \%$ in 2002 , marking the Index's sixth negative annual return in our 16-year period (Figures 8 and 9). The Index experienced negative returns in seven of the 12 months in 2002, with significant negative returns in June and July. The relatively poor results are not surprising as the supply of newly defaulted bond issues increased throughout 2002, helping to drive down prices. ${ }^{3}$ Monthly returns for all 16 years of the A-NYU Bond Index are listed in Appendix A. The level of the Index decreased from 194.1 at the end of 2001 to 182.5 at the end of 2002 (December $1986=100)$.

In 2002, the market experienced seven months with negative returns, but only one of these negative monthly returns was in excess of $5 \%$ ( $-9.44 \%$ in June). The June/July period was a particularly difficult one for most risky securities markets. The S\&P 500 Stock Index, which finished with an annual return of $-22.08 \%$ (assuming reinvestment of dividends) in 2002, was comparatively volatile and experienced seven months with negative returns in excess of $5 \%$ (see Figure 10 for a listing of comparative returns for highly volatile $( \pm 5 \%)$ stock market months).

Defaulted bond securities outperformed the total return on the S\&P 500 Stock Index for the second year in a row. The Defaulted Bond Index was outperformed by the

\footnotetext{
${ }^{3}$ See Altman, Brady, Resti and Sironi (2002) for a detailed analysis of the supply/demand dynamic and its impact on corporate debt prices just after default.
} 
Salomon Smith Barney High Yield Bond Market Index, which returned -1.53\%. Tenyear government bonds outperformed all of our risky security indexes posting a positive return of $14.66 \%$.

\section{Sixteen-Year Comparative Performance}

Figure 8 exhibits the return on defaulted bonds, common stocks, and high yield bonds over the entire sixteen-year sample period, 1987-2002. The arithmetic annual average for the Altman-NYU Salomon Center Defaulted Bond Index (6.27\% per year) is about half of that of the S\&P 500 Stock Index (12.58\% per year) and below that of the Salomon Smith Barney High Yield Bond Market Index (8.47\% per year) for the sample period. In seven of the 16 years, defaulted bonds performed worse than both of the other two indexes, while in six years our bond Index was the best performer.

The standard deviation of annual returns for the defaulted bond index decreased slightly in 2002, but it still remains the highest of the three indexes. Comparing volatility on a monthly basis, however, the standard deviation of monthly returns for defaulted bond issues $(4.30 \%)$ is, in fact, lower than that of the S\&P 500 Stock Index $(4.66 \%)$ while both of these indexes are considerably more volatile than the high yield bond index $(2.15 \%)$. The discrepancy between the standard deviations of high yield bonds and defaulted bonds is consistent with high yield bonds paying a fairly steady fixed interest component and defaulted bonds paying no interest.

\section{Defaulted Bank Loan Performance}

Today's managers of distressed securities are more commonly investing in both distressed bonds and the private debt (particularly bank debt) of defaulting companies. The observed increasing investment in defaulted private debt has been coincident with 
the bank loan market's increasing size and liquidity as market makers have devoted considerable resources to bank debt trading.

In 2002, our Bank Loan Index performed relatively well compared to most asset classes, returning 3.03\% for the year and closing at 120.5 (December 1995=100). The Index outperformed our Defaulted Bond Index as well as both the S\&P 500 Index and the Salomon Smith Barney High Yield Bond Market Index (Figure 11). Defaulted bank loans are considerably less volatile. Our Bank Loan Index experienced two abnormally low monthly returns, July and August, when it fell by more than $4 \%$ each month and six months overall of negative returns. Like the Bond Index, November was an excellent month for returns. Appendix B shows the performance of our Defaulted Bank Loan Index from its inception through December 31, 2002.

The average annual return of the Defaulted Bank Loan Index since its inception in 1996 dropped slightly from $3.18 \%$ as of the end of 2001 to 3.16 in 2002 . It remains above the average annual return for the Defaulted Bond Index over the comparable period but still trails both the equity and high yield bond indexes (Figure 11).

\section{Combined Bond and Bank Loan Index}

Our Combined Defaulted Securities Index is calculated based on the combined market values and total returns of public bonds and private bank loans. The Index, from its inception in 1996 through 2002, is displayed in Appendix C. The annual return for the Combined Index was $-0.53 \%$ for 2002 . This cumulative index level closed out the year at 96.7 , down slightly from 97.2 in 2001 . The Combined Index enables us to benchmark performance criteria for a more broadly defined defaulted securities market. 


\section{Diversification: Management Styles and Return Correlations}

One strategy that our analysis will suggest is to include defaulted debt in a larger portfolio of risky securities. Several domestic pension funds and foreign portfolios have effectively used this strategy by allocating a portion of their total investments to defaulted debt money managers. The principal idea for this strategy is that the returns from investing in distressed debt securities have relatively low correlations with most other major asset classes. This can be clearly seen from the data on returns that we have been tracking for many years.

Figure 12 exhibits the correlation between the Altman-NYU Defaulted Bond Index and each of the two other risky asset classes - common stocks and high yield bonds - for the last sixteen years. As of December 31,2002, we observe that the monthly return correlation between defaulted debt and the S\&P 500 Stock Index is only $27.59 \%$. The correlation between defaulted bonds and S\&P equities is slightly above the correlation between these two asset classes as of last year $(24.89 \%)$. The low correlation is important to note because holders of defaulted debt usually exchange their debt for the equity of the emerged Chapter 11 entity, unless they sell the debt just prior to emergence. The correlation between these two asset classes on a quarterly basis is slightly higher at $32.6 \%$.

The correlation between defaulted bonds and high yield bonds, however, is comparatively high. The monthly correlation of returns is $60.16 \%$, while the quarterly correlation between these two asset classes is $58.10 \%$. As was the case in 2001 , the correlation between high yield bonds and the Defaulted Loan Index (see Figure 13) is lower than that of defaulted bonds and high yield bonds, at $41.77 \%$ and $40.18 \%$ for 
monthly and quarterly returns, respectively. The returns for defaulted bank loans have an inverse relationship with the S\&P 500 as the correlation is $-5.77 \%$. Finally, the monthly returns correlation between our two defaulted debt indexes (bonds and bank loans) was “only" 56.93\%. One might expect a somewhat higher correlation.

\section{Diversification by Manager Style}

Almost all portfolio managers involved in the distressed market have been specialists in the sector, rather than investors in distressed bonds within broader-based portfolios. Therefore, the avenue of diversification appears to be primarily through the use of different investment managers (there are some rare exceptions where a fund combines investments in more traditional debt and equity securities combined with distressed securities). Some "fund-of-funds" and foreign closed-end Funds have adopted the strategy of selecting managers of distressed securities with different styles. In addition to diversifying across asset classes, these funds have a strategy of investing with managers of distressed securities who practice different approaches (e.g., active, passive, control, long-short, senior vs. subordinate). A partial list of almost 60 distressed debt investors can be found in Appendix D and a listing of three major types of strategies and their sub-strategies is given in Appendix E.

\section{Bank Loan Recovery Analysis}

Due to the significant increase in the number of defaulted loan facilities and the attendant interest from investors, there is an increasing number of published studies on recovery rates on defaulted bank loans (e.g., from the rating agencies). Also, with the continued disparity between our Bank Loan Index's market/face value ratio and the typical average experience, we believe that estimating recovery rates on defaulted bank 
loans using our Index will be interesting. Our analysis of bank loan recoveries contained a sample of 262 defaulted bank loan facilities from 1996 through 2002. The recovery rate is determined by prices or price quotes from the secondary market at or just after default.

We discovered that the median, weighted average and arithmetic average values of defaulted bank loan recovery rates over this period were $70.54 \%, 69.02 \%$, and $69.22 \%$ respectively (Figure 14). The standard error was $19.62 \%$, which somewhat supports a wide dispersion of defaulted bank loan recovery rates, including a number of particularly low rates. We also observed recovery rates by year of default and found that both the median and mean defaulted bank loan recovery values have generally decreased over time. This was particularly true for the weighted average, which fell from the $80-90 \%$ range in $1996-1998$ to the $52-59 \%$ range in $1999-2002$. It is interesting to note that median and arithmetic average recoveries increased in 2002 vis-à-vis 2001, but the weighted (by size) average decreased to $52 \%$.

In addition, we attempted to determine the extent to which defaulted bank loan recovery rates for telecommunications and e-commerce companies mirrored the experience of defaulted bonds for companies in those sectors, which were considerably lower than those of companies in more traditional sectors. Figure 15 displays the results for telecommunications and e-commerce companies only for the more relevant 19992002 period. It is interesting to note that median, mean and weighted mean recoveries are only marginally lower and do not have the same experience as recovery rates on defaulted bonds in these sectors. The latter were extremely low (under 20\%) for the comparable period (see Figure 19 of our other 2002 report). 
As expected, these results compare favorably (i.e., are higher) with the recovery rates of defaulted bonds. Average loan recoveries in recent years, however, are much below the historical average as reflected in the market/face value ratio. 


\section{References for this Study}

Altman Foothill Report, 1999, Investing in Distressed Securities, Foothill Corporation, Los Angeles, April, and The Market for Distressed Securities and Bank Loans, 1992.

Altman, E. I. \& A. Eberhart, 1994, "Do Seniority Provisions Protect Bondholders' Investments?"Journal of Portfolio Management, Summer.

Altman, E. I., B. Brady, A. Resti \& A. Sironi, 2001, “The Link Between Default and Recovery Rates," NYU Salomon Center, WP \#S-02-9 and ISDA, January.

Altman, E. I., and P. Arman, 2002, "Defaults and Returns in the High Yield Bond Market: Analysis through 2001," NYU Salomon Center Working Paper S-02-4, January.

Altman, E. I., and J. Pompeii, 2002, "Market Size and Investment Performance of Defaulted Bonds and Bank Loans: 1987-2001," NYU Salomon Center Working Paper S-02-5, January.

Brand, L. and R. Behar, 2000, "Recoveries on Defaulted Bonds Tied to Security Ratings,” S\&P's Credit Week February.

Caouette, J., E. Altman and P. Narayanan (1998), Managing Credit Risk: The Next Great Financial Challenge, John Wiley \& Sons, New York.

Fitch, 2001, “Bank Loan and Bond Recovery Study: 1997-2001,” S. O’Shea, S. Bonelli, and R. Grossman, March 19.

Fitch, 1997, “Syndicated Bank Loan Recovery Study,” R. Grossman, M. Brennan and Vento, October 22.

Keisman, D., 2003, “Update on Recovery Rates,” S\&P, February.

Hamilton, D., G. Gupta and A. Berthault, 2001, "Default and Recovery Rates of Corporate Bond Issuers: 2000,” Moody’s, February. 
FIGURE 1

\section{HISTORICAL DEFAULT RATES - STRAIGHT BONDS ONLY EXCLUDING DEFAULTED ISSUES FROM PAR VALUE OUTSTANDING 1971 - 2002 (\$ MILLIONS)}

\begin{tabular}{|c|c|c|c|c|}
\hline YEAR & $\begin{array}{c}\text { PAR VALUE } \\
\text { OUTSTANDING (a) }\end{array}$ & $\begin{array}{r}\text { PAR VALUE } \\
\text { DEFAULTS }\end{array}$ & $\begin{array}{l}\text { DEFAULT } \\
\text { RATES }\end{array}$ & \\
\hline 2002 & $\$ 757,000$ & $\$ 96,858$ & $12.795 \%$ & \\
\hline 2001 & $\$ 649,000$ & $\$ 63,609$ & $9.801 \%$ & \\
\hline 2000 & $\$ 597,200$ & $\$ 30,295$ & $5.073 \%$ & \\
\hline 1999 & $\$ 567,400$ & $\$ 23,532$ & $4.147 \%$ & \\
\hline 1998 & $\$ 465,500$ & $\$ 7,464$ & $1.603 \%$ & \\
\hline 1997 & $\$ 335,400$ & $\$ 4,200$ & $1.252 \%$ & \\
\hline 1996 & $\$ 271,000$ & $\$ 3,336$ & $1.231 \%$ & \\
\hline 1995 & $\$ 240,000$ & $\$ 4,551$ & $1.896 \%$ & \\
\hline 1994 & $\$ 235,000$ & $\$ 3,418$ & $1.454 \%$ & \\
\hline 1993 & $\$ 206,907$ & $\$ 2,287$ & $1.105 \%$ & \\
\hline 1992 & $\$ 163,000$ & $\$ 5,545$ & $3.402 \%$ & \\
\hline 1991 & $\$ 183,600$ & $\$ 18,862$ & $10.273 \%$ & \\
\hline 1990 & $\$ 181,000$ & $\$ 18,354$ & $10.140 \%$ & \\
\hline 1989 & $\$ 189,258$ & $\$ 8,110$ & $4.285 \%$ & \\
\hline 1988 & $\$ 148,187$ & $\$ 3,944$ & $2.662 \%$ & \\
\hline 1987 & $\$ 129,557$ & $\$ 7,486$ & $5.778 \%$ & \\
\hline 1986 & $\$ 90,243$ & $\$ 3,156$ & $3.497 \%$ & \\
\hline 1985 & $\$ 58,088$ & $\$ 992$ & $1.708 \%$ & \\
\hline 1984 & $\$ 40,939$ & $\$ 344$ & $0.840 \%$ & \\
\hline 1983 & $\$ 27,492$ & $\$ 301$ & $1.095 \%$ & \\
\hline 1982 & $\$ 18,109$ & $\$ 577$ & $3.186 \%$ & \\
\hline 1981 & $\$ 17,115$ & $\$ 27$ & $0.158 \%$ & \\
\hline 1980 & $\$ 14,935$ & $\$ 224$ & $1.500 \%$ & \\
\hline 1979 & $\$ 10,356$ & $\$ 20$ & $0.193 \%$ & \\
\hline 1978 & $\$ 8,946$ & $\$ 119$ & $1.330 \%$ & \\
\hline 1977 & $\$ 8,157$ & $\$ 381$ & $4.671 \%$ & \\
\hline 1976 & $\$ 7,735$ & $\$ 30$ & $0.388 \%$ & \\
\hline 1975 & $\$ 7,471$ & $\$ 204$ & $2.731 \%$ & \\
\hline 1974 & $\$ 10,894$ & $\$ 123$ & $1.129 \%$ & \\
\hline 1973 & $\$ 7,824$ & $\$ 49$ & $0.626 \%$ & \\
\hline 1972 & $\$ 6,928$ & $\$ 193$ & $2.786 \%$ & \\
\hline 1971 & $\$ 6,602$ & $\$ 82$ & $1.242 \%$ & $\begin{array}{l}\text { Standard } \\
\text { Deviation }\end{array}$ \\
\hline \multirow{3}{*}{\multicolumn{2}{|c|}{ ARITHMETIC AVERAGE DEFAULT RATE }} & 1971 TO 2002 & $3.249 \%$ & $3.201 \%$ \\
\hline & & $1978 \mathrm{TO} 2002$ & $3.616 \%$ & $3.455 \%$ \\
\hline & & 1985 TO 2002 & $4.561 \%$ & $3.611 \%$ \\
\hline \multirow{2}{*}{\multicolumn{2}{|c|}{ WEIGHTED AVERAGE DEFAULT RATE (b) }} & $\begin{array}{l}1971 \text { TO } 2002 \\
1978 \text { TO } 2002\end{array}$ & $\begin{array}{l}5.453 \% \\
5.488 \%\end{array}$ & \\
\hline & & 1985 TO 2002 & $5.597 \%$ & \\
\hline MEDIAN ANNUA & $\overline{\mathbf{A T E}}$ & 1971 TO 2002 & $1.802 \%$ & \\
\hline
\end{tabular}

Notes

(a) As of mid-year.

(b) Weighted by par value of amount outstanding for each year. 
FIGURE 2

DISTRESSED (a) AND DEFAULTED DEBT AS A PERCENTAGE OF TOTAL HIGH YIELD DEBT MARKET

1990-2002 ${ }^{(b)}$

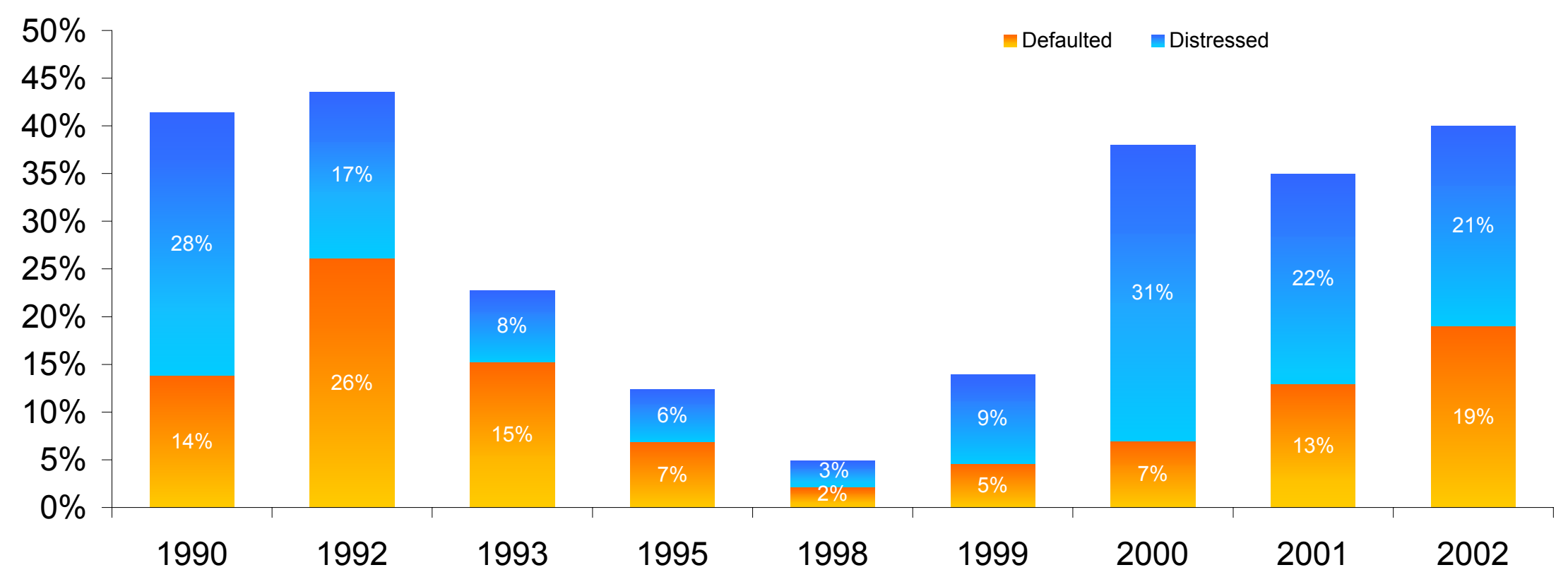

(a) Defined as yield-to-maturity spread greater than or equal to $1000 \mathrm{bp}$ over comparable Treasuries

(b) Some years not available as no survey results available

Source: Salomon Smith Barney and NYU Salomon Center 
FIGURE 3

\section{ESTIMATED FACE AND MARKET VALUES OF DEFAULTED AND DISTRESSED DEBT 2001-2002 (\$ billion)}

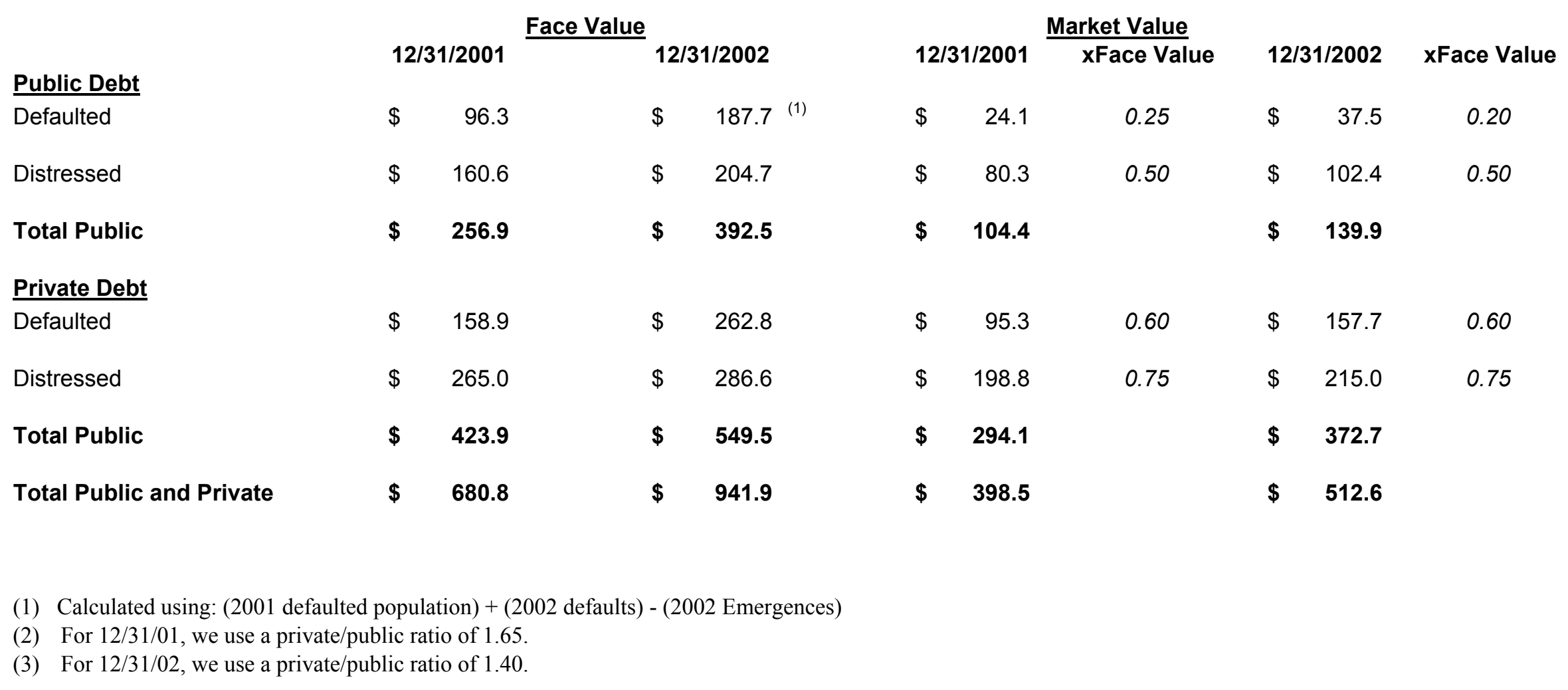

Sources: Estimated by Professor Edward Altman, NYU Stern School of Business from Salomon Smith Barney's High Yield Bond Database, NYU Salomon Center Defaulted Bond and Bank Loan Databases. 


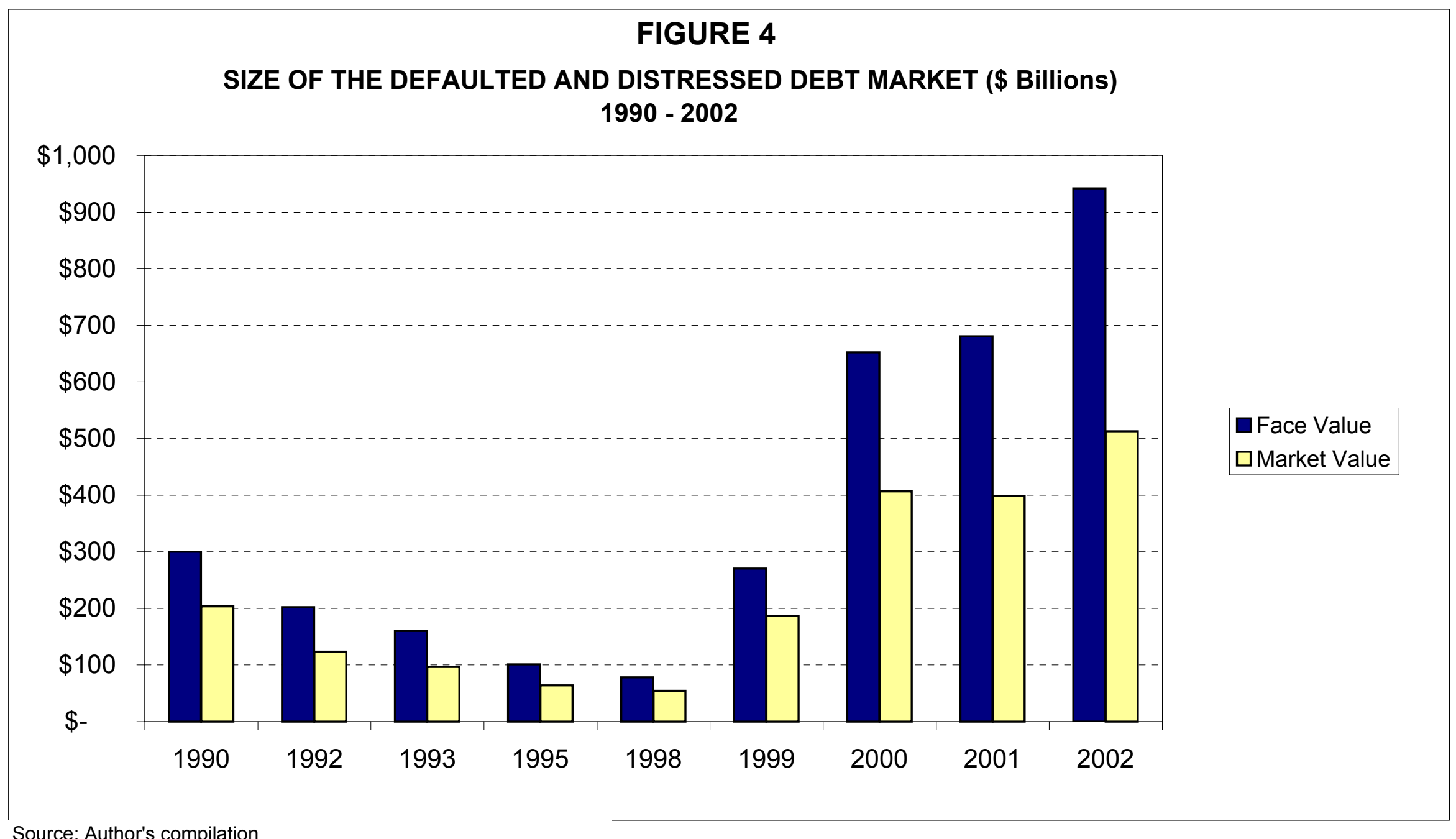

Source: Author's compilation 


\section{FIGURE 5}

\section{SIZE OF THE ALTMAN-NYU SALOMON CENTER DEFAULTED BOND INDEX \\ (1986 - 2002)}

\begin{tabular}{cccccc}
\hline $\begin{array}{c}\text { Year } \\
\text { End }\end{array}$ & $\begin{array}{c}\text { Number of } \\
\text { Issues }\end{array}$ & $\begin{array}{c}\text { Number of } \\
\text { Firms }\end{array}$ & $\begin{array}{c}\text { Face Value } \\
\text { (\$ Billions) }\end{array}$ & $\begin{array}{c}\text { Market Value } \\
\text { (\$ Billions) }\end{array}$ & $\begin{array}{c}\text { Market / } \\
\text { Face Ratio }\end{array}$ \\
\hline 1986 & 30 & 10 & 1.7 & 0.5 & 0.29 \\
1987 & 53 & 18 & 5.7 & 4.2 & 0.74 \\
1988 & 91 & 34 & 5.2 & 2.7 & 0.52 \\
1989 & 111 & 35 & 8.7 & 3.4 & 0.39 \\
1990 & 173 & 68 & 18.7 & 5.1 & 0.27 \\
1991 & 207 & 80 & 19.6 & 6.1 & 0.31 \\
1992 & 231 & 90 & 21.7 & 11.1 & 0.51 \\
1993 & 151 & 77 & 11.8 & 5.8 & 0.49 \\
1994 & 93 & 35 & 6.3 & 3.3 & 0.52 \\
1995 & 50 & 27 & 5.0 & 2.3 & 0.46 \\
1996 & 39 & 28 & 5.3 & 2.4 & 0.45 \\
1997 & 37 & 26 & 5.9 & 2.7 & 0.46 \\
1998 & 36 & 30 & 5.5 & 1.4 & 0.25 \\
1999 & 83 & 60 & 16.3 & 4.1 & 0.25 \\
2000 & 129 & 72 & 27.8 & 4.3 & 0.15 \\
2001 & 202 & 86 & 56.2 & 11.8 & 0.21 \\
2002 & 166 & 113 & 61.6 & 10.4 & 0.17 \\
\hline
\end{tabular}

Source: Altman-NYU Salomon Center Defaulted Bond and Bank Loan Databases 
FIGURE 6

\section{SIZE OF THE ALTMAN-NYU SALOMON CENTER DEFAULTED BANK LOAN INDEX \\ (1995 - 2002)}

\begin{tabular}{cccccc}
\hline $\begin{array}{c}\text { Year } \\
\text { End }\end{array}$ & $\begin{array}{c}\text { Number of } \\
\text { Issues }\end{array}$ & $\begin{array}{c}\text { Number of } \\
\text { Firms }\end{array}$ & $\begin{array}{c}\text { Face Value } \\
\text { (\$ Billions) }\end{array}$ & $\begin{array}{c}\text { Market Value } \\
\text { (\$ Billions) }\end{array}$ & $\begin{array}{c}\text { Market/ } \\
\text { Face Ratio }\end{array}$ \\
\hline 1995 & 17 & 14 & 2.9 & 2.0 & 0.69 \\
1996 & 23 & 22 & 4.2 & 3.3 & 0.79 \\
1997 & 18 & 15 & 3.4 & 2.4 & 0.71 \\
1998 & 15 & 13 & 3.0 & 1.9 & 0.63 \\
1999 & 45 & 23 & 12.9 & 6.8 & 0.53 \\
2000 & 100 & 39 & 26.9 & 13.6 & 0.51 \\
2001 & 141 & 56 & 44.7 & 23.8 & 0.53 \\
2002 & 64 & 51 & 37.7 & 17.4 & 0.46 \\
\hline
\end{tabular}

Source: Altman-NYU Salomon Center Defaulted Bond and Bank Loan Databases 


\section{FIGURE 7}

Defaulted Debt Indexes: Market-to-Face Value Ratio

(Annual 1986 - 2002)

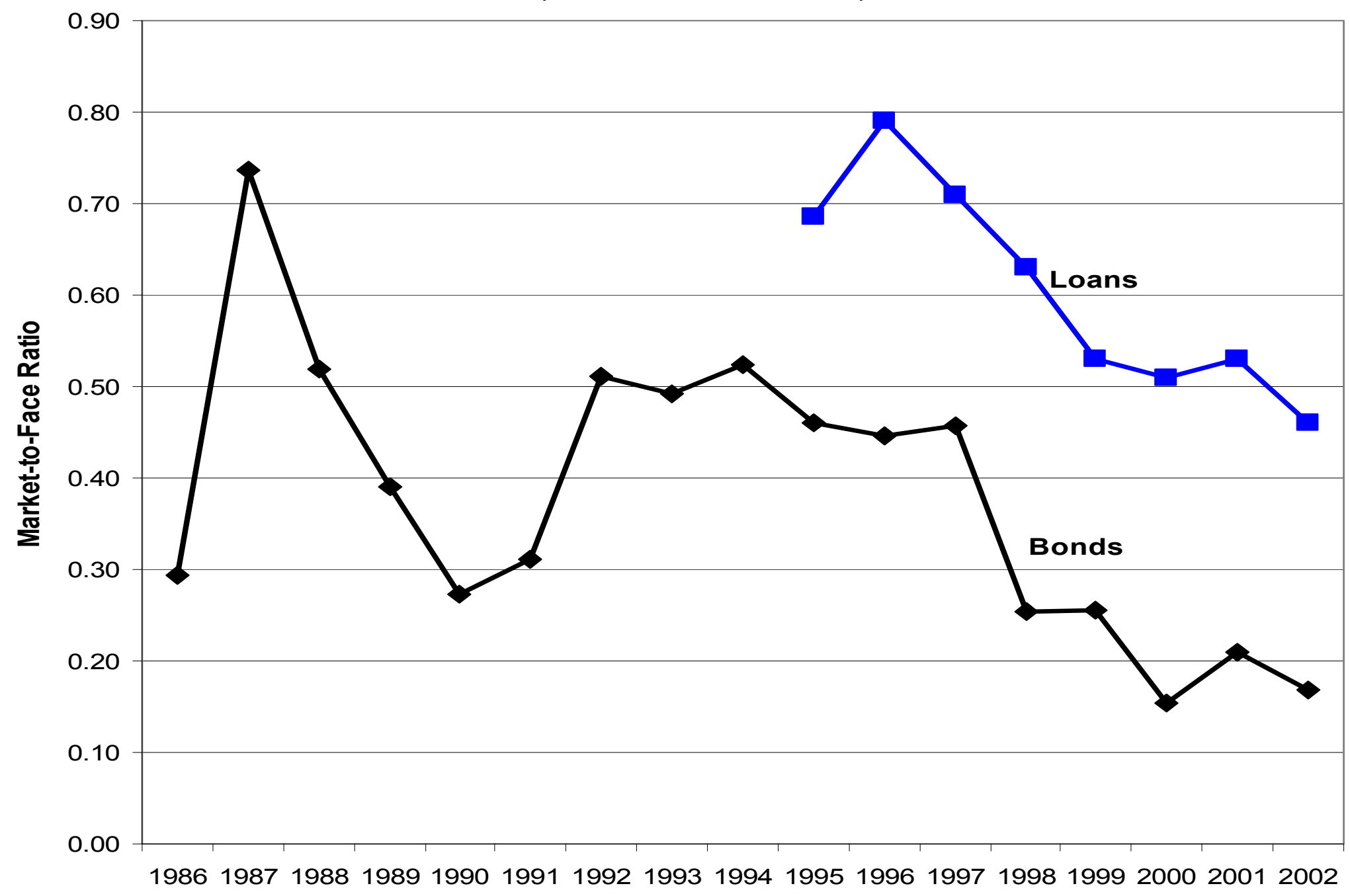

Source: Figures 5 and 6 


\section{FIGURE 8 \\ ALTMAN-NYU SALOMON CENTER \\ DEFAULTED BOND INDEX \\ COMPARISON OF RETURNS \\ (1987 - 2002)}

\begin{tabular}{|c|c|c|c|}
\hline Year & $\begin{array}{c}\text { Altman-NYU Salomon Center } \\
\text { Defaulted Bond Index }\end{array}$ & S\&P 500 Stock Index & $\begin{array}{c}\text { Salomon Smith Barney High } \\
\text { Yield Market Index }\end{array}$ \\
\hline 1987 & $37.85 \%$ & $5.26 \%$ & $4.67 \%$ \\
\hline 1988 & $26.49 \%$ & $16.61 \%$ & $13.47 \%$ \\
\hline 1989 & $-22.78 \%$ & $31.68 \%$ & $2.75 \%$ \\
\hline 1990 & $-17.08 \%$ & $-3.12 \%$ & $-7.04 \%$ \\
\hline 1991 & $43.11 \%$ & $30.48 \%$ & $39.93 \%$ \\
\hline 1992 & $15.39 \%$ & $7.62 \%$ & $17.86 \%$ \\
\hline 1993 & $27.91 \%$ & $10.08 \%$ & $17.36 \%$ \\
\hline 1994 & $6.66 \%$ & $1.32 \%$ & $-1.25 \%$ \\
\hline 1995 & $11.26 \%$ & $37.56 \%$ & $19.71 \%$ \\
\hline 1996 & $10.21 \%$ & $22.96 \%$ & $11.29 \%$ \\
\hline 1997 & $-1.58 \%$ & $34.36 \%$ & $13.18 \%$ \\
\hline 1998 & $-26.91 \%$ & $28.58 \%$ & $3.60 \%$ \\
\hline 1999 & $11.34 \%$ & $20.98 \%$ & $1.74 \%$ \\
\hline 2000 & $-33.09 \%$ & $-9.11 \%$ & $-5.68 \%$ \\
\hline 2001 & $17.47 \%$ & $-11.87 \%$ & $5.44 \%$ \\
\hline 2002 & $-5.98 \%$ & $-22.08 \%$ & $-1.53 \%$ \\
\hline \multicolumn{4}{|l|}{1987 - 2002 Arithmetic } \\
\hline Average (Annual) Rate & $6.27 \%$ & $12.58 \%$ & $8.47 \%$ \\
\hline Standard Deviation & $22.74 \%$ & $18.19 \%$ & $11.83 \%$ \\
\hline 1987 - 2002 Compounded & $3.83 \%$ & $11.13 \%$ & $7.90 \%$ \\
\hline \multicolumn{4}{|l|}{ Average (Annual) Rate } \\
\hline \multicolumn{4}{|l|}{1987 - 2002 Arithmetic } \\
\hline Average (Monthly) Rate & $0.41 \%$ & $0.99 \%$ & $0.66 \%$ \\
\hline Standard Deviation & $4.30 \%$ & $4.66 \%$ & $2.14 \%$ \\
\hline 1987 - 2002 Compounded & $0.27 \%$ & $0.82 \%$ & $0.62 \%$ \\
\hline Average (Monthly) Rate & & & \\
\hline
\end{tabular}

Source: Altman-NYU Salomon Center Defaulted Bond and Bank Loan Databases 


\section{FIGURE 9}

\section{DEFAULTED BOND, STOCK, AND HIGH YIELD BOND INDICES}

1987-2002

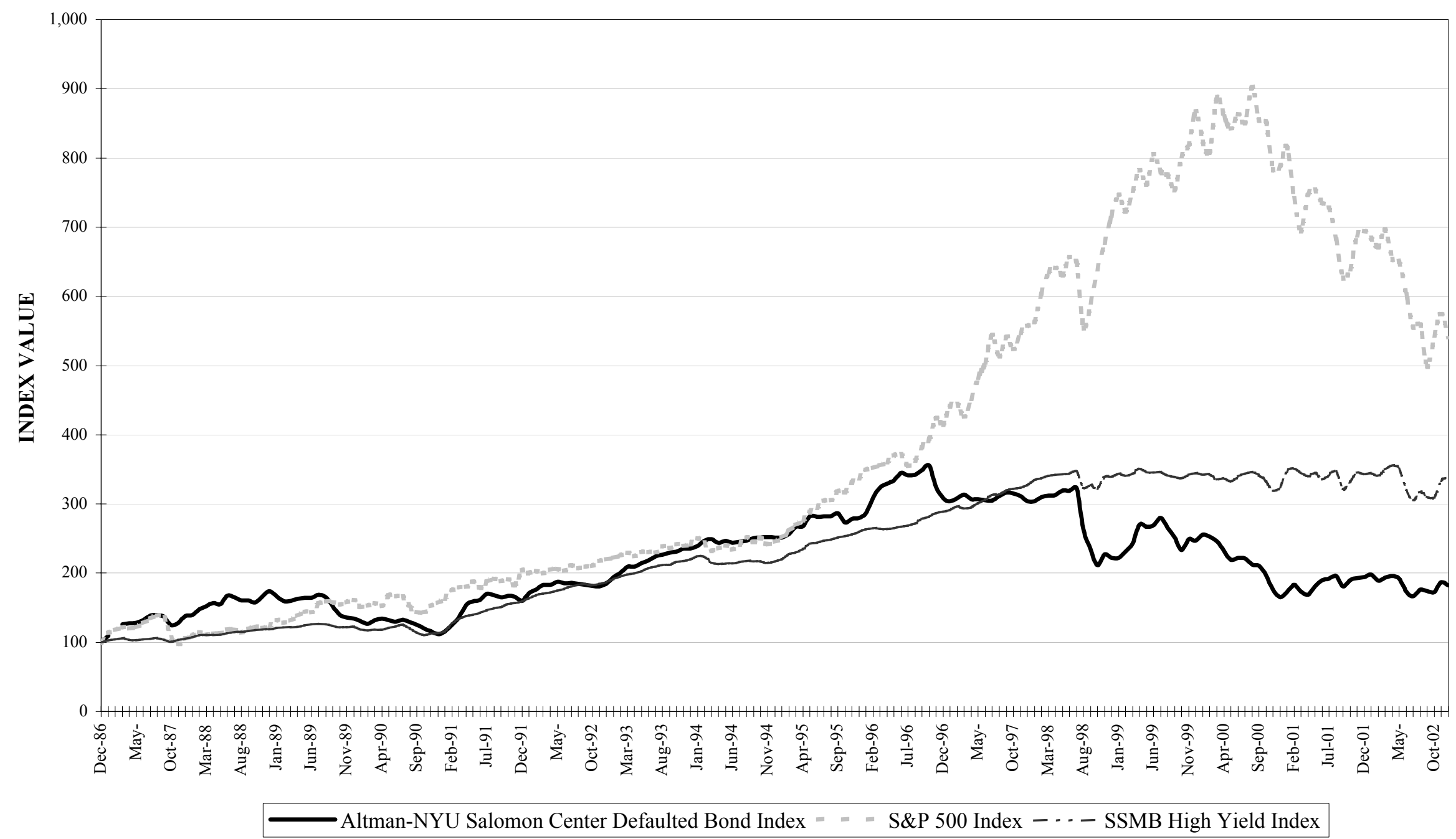

Source: Figure 8 
FIGURE 10

COMPARISON BETWEEN INDEX RETURNS

GIVEN A CHANGE IN S\&P GREATER THAN 5\%

\begin{tabular}{|c|c|c|c|c|}
\hline Month & $\begin{array}{l}\text { Bank Loan } \\
\text { Index }\end{array}$ & Bond Index & $\begin{array}{c}\text { S\&P } 500 \\
\text { Stock Index }\end{array}$ & $\begin{array}{c}\text { Salomon Smith Barney } \\
\text { High Yield Index }\end{array}$ \\
\hline Jan-87 & & $9.80 \%$ & $13.47 \%$ & $2.83 \%$ \\
\hline Jun-87 & & $2.90 \%$ & $5.05 \%$ & $1.38 \%$ \\
\hline Jul-87 & & $5.50 \%$ & $5.07 \%$ & $0.54 \%$ \\
\hline Oct-87 & & $-8.92 \%$ & $-21.54 \%$ & $-2.67 \%$ \\
\hline Nov-87 & & $3.22 \%$ & $-8.24 \%$ & $2.53 \%$ \\
\hline Dec-87 & & $7.53 \%$ & $7.61 \%$ & $1.33 \%$ \\
\hline Jan-89 & & $-4.47 \%$ & $7.32 \%$ & $1.50 \%$ \\
\hline Apr-89 & & $2.06 \%$ & $5.19 \%$ & $0.30 \%$ \\
\hline Jul-89 & & $2.46 \%$ & $9.03 \%$ & $0.47 \%$ \\
\hline Jan-90 & & $-2.91 \%$ & $-6.71 \%$ & $-3.03 \%$ \\
\hline May-90 & & $-1.23 \%$ & $9.75 \%$ & $2.63 \%$ \\
\hline Aug-90 & & $-3.03 \%$ & $-9.04 \%$ & $-3.87 \%$ \\
\hline Nov-90 & & $-2.69 \%$ & $6.46 \%$ & $2.02 \%$ \\
\hline Feb-91 & & $8.49 \%$ & $7.15 \%$ & $8.82 \%$ \\
\hline Dec-91 & & $-3.53 \%$ & $11.44 \%$ & $1.34 \%$ \\
\hline Sep-96 & $0.79 \%$ & $2.11 \%$ & $5.63 \%$ & $2.34 \%$ \\
\hline Nov-96 & $0.37 \%$ & $-8.62 \%$ & $7.56 \%$ & $1.92 \%$ \\
\hline Jan-97 & $1.88 \%$ & $-1.54 \%$ & $6.25 \%$ & $0.75 \%$ \\
\hline Apr-97 & $-6.63 \%$ & $-2.13 \%$ & $5.97 \%$ & $0.71 \%$ \\
\hline May-97 & $-1.93 \%$ & $0.11 \%$ & $6.88 \%$ & $2.02 \%$ \\
\hline Jul-97 & $0.45 \%$ & $-0.23 \%$ & $7.96 \%$ & $2.29 \%$ \\
\hline Aug-97 & $1.19 \%$ & $2.27 \%$ & $-5.60 \%$ & $0.25 \%$ \\
\hline Sep-97 & $2.41 \%$ & $1.64 \%$ & $5.48 \%$ & $1.75 \%$ \\
\hline Feb-98 & $-0.84 \%$ & $1.96 \%$ & $7.21 \%$ & $0.68 \%$ \\
\hline
\end{tabular}


FIGURE 10

COMPARISON BETWEEN INDEX RETURNS

GIVEN A CHANGE IN S\&P GREATER THAN 5\%

\begin{tabular}{|c|c|c|c|c|}
\hline Month & $\begin{array}{l}\text { Bank Loan } \\
\text { Index }\end{array}$ & Bond Index & $\begin{array}{c}\text { S\&P 500 } \\
\text { Stock Index }\end{array}$ & $\begin{array}{c}\text { Salomon Smith Barney } \\
\text { High Yield Index }\end{array}$ \\
\hline Mar-98 & $1.68 \%$ & $0.82 \%$ & $5.12 \%$ & $1.08 \%$ \\
\hline Aug-98 & $-6.26 \%$ & $-18.25 \%$ & $-14.46 \%$ & $-6.70 \%$ \\
\hline Sep-98 & $-6.16 \%$ & $-11.21 \%$ & $6.41 \%$ & $1.23 \%$ \\
\hline Oct-98 & $-7.88 \%$ & $-9.48 \%$ & $8.13 \%$ & $-1.38 \%$ \\
\hline Nov-98 & $5.44 \%$ & $7.32 \%$ & $6.06 \%$ & $5.02 \%$ \\
\hline Dec-98 & $-0.85 \%$ & $-2.43 \%$ & $5.76 \%$ & $-0.07 \%$ \\
\hline Jun-99 & $2.58 \%$ & $1.00 \%$ & $5.50 \%$ & $-0.22 \%$ \\
\hline Oct-99 & $-2.64 \%$ & $-7.13 \%$ & $6.33 \%$ & $-0.68 \%$ \\
\hline Dec-99 & $-0.12 \%$ & $-0.92 \%$ & $5.89 \%$ & $0.84 \%$ \\
\hline Jan-00 & $3.64 \%$ & $3.50 \%$ & $-5.02 \%$ & $-0.83 \%$ \\
\hline Mar-00 & $-5.48 \%$ & $-2.86 \%$ & $9.77 \%$ & $-2.03 \%$ \\
\hline Aug-00 & $-0.65 \%$ & $-3.96 \%$ & $6.21 \%$ & $0.74 \%$ \\
\hline Sep-00 & $0.86 \%$ & $-0.64 \%$ & $-5.28 \%$ & $-1.07 \%$ \\
\hline Nov-00 & $-1.39 \%$ & $-10.10 \%$ & $-7.88 \%$ & $-4.09 \%$ \\
\hline Feb-01 & $6.71 \%$ & $5.90 \%$ & $-9.11 \%$ & $1.19 \%$ \\
\hline Mar-01 & $0.57 \%$ & $-5.43 \%$ & $-6.33 \%$ & $-1.82 \%$ \\
\hline Apr-01 & $-2.31 \%$ & $-2.57 \%$ & $7.76 \%$ & $-1.47 \%$ \\
\hline Aug-01 & $3.05 \%$ & $2.04 \%$ & $-6.25 \%$ & $1.37 \%$ \\
\hline Sep-01 & $-1.99 \%$ & $-7.84 \%$ & $-8.07 \%$ & $-7.20 \%$ \\
\hline Nov-01 & $1.12 \%$ & $1.57 \%$ & $7.67 \%$ & $3.80 \%$ \\
\hline Apr-02 & $3.27 \%$ & $1.29 \%$ & $-6.06 \%$ & $1.64 \%$ \\
\hline Jun-02 & $-1.64 \%$ & $-9.44 \%$ & $-7.12 \%$ & $-8.81 \%$ \\
\hline Jul-02 & $-4.27 \%$ & $-4.19 \%$ & $-7.79 \%$ & $-4.52 \%$ \\
\hline Sep-02 & $-2.03 \%$ & $-1.11 \%$ & $-10.86 \%$ & $-1.97 \%$ \\
\hline
\end{tabular}


FIGURE 10

COMPARISON BETWEEN INDEX RETURNS

GIVEN A CHANGE IN S\&P GREATER THAN 5\%

\begin{tabular}{rcccc}
\hline Month & $\begin{array}{c}\text { Bank Loan } \\
\text { Index }\end{array}$ & Bond Index & $\begin{array}{c}\text { S\&P 500 } \\
\text { Stock Index }\end{array}$ & $\begin{array}{c}\text { Salomon Smith Barney } \\
\text { High Yield Index }\end{array}$ \\
\hline Oct-02 & $-1.25 \%$ & $-1.27 \%$ & $8.80 \%$ & $-0.34 \%$ \\
Nov-02 & $5.37 \%$ & $8.49 \%$ & $5.88 \%$ & $7.63 \%$ \\
Dec-02 & $2.51 \%$ & $-2.28 \%$ & $-5.87 \%$ & $1.21 \%$ \\
\hline
\end{tabular}

Source: Altman-NYU Salomon Center Defaulted Bond and Bank Loan Databases 


\section{FIGURE 11 \\ ALTMAN-NYU SALOMON CENTER \\ BANK LOAN INDEX \\ COMPARISON OF RETURNS \\ (1996 - 2002)}

\begin{tabular}{|c|c|c|c|}
\hline Year & $\begin{array}{c}\text { Altman-NYU Salomon } \\
\text { Center Bank Loan } \\
\text { Index } \\
\end{array}$ & $\begin{array}{c}\text { S\&P } 500 \text { Stock } \\
\text { Index }\end{array}$ & $\begin{array}{c}\text { Salomon Smith Barney } \\
\text { High Yield Market Index } \\
\end{array}$ \\
\hline 1996 & $19.56 \%$ & $22.96 \%$ & $11.29 \%$ \\
\hline 1997 & $1.75 \%$ & $34.36 \%$ & $13.18 \%$ \\
\hline 1998 & $-10.22 \%$ & $28.58 \%$ & $3.60 \%$ \\
\hline 1999 & $0.65 \%$ & $20.98 \%$ & $1.74 \%$ \\
\hline 2000 & $-6.59 \%$ & $-9.11 \%$ & $-5.68 \%$ \\
\hline 2001 & $13.94 \%$ & $-11.87 \%$ & $5.44 \%$ \\
\hline 2002 & $3.03 \%$ & $-22.08 \%$ & $-1.53 \%$ \\
\hline 1996 - Dec 2002 Arithmetic & & & \\
\hline Average (Annual) Rate & $3.16 \%$ & $9.12 \%$ & $4.01 \%$ \\
\hline Standard Deviation & $10.55 \%$ & $22.71 \%$ & $6.70 \%$ \\
\hline $\begin{array}{l}1996 \text { - Dec } 2002 \text { Compounded } \\
\text { Average (Annual) Rate }\end{array}$ & $2.70 \%$ & $6.98 \%$ & $3.82 \%$ \\
\hline 1996 - Dec 2002 Arithmetic & & & \\
\hline Average (Monthly) Rate & $0.26 \%$ & $0.70 \%$ & $0.34 \%$ \\
\hline Standard Deviation & $2.85 \%$ & $5.13 \%$ & $2.47 \%$ \\
\hline $\begin{array}{l}1996 \text { - Dec } 2002 \text { Compounded } \\
\text { Average (Monthly) Rate }\end{array}$ & $0.21 \%$ & $0.52 \%$ & $0.31 \%$ \\
\hline
\end{tabular}

Source: Altman-NYU Salomon Center Defaulted Bond and Bank Loan Databases 


\section{FIGURE 12}

\section{CORRELATION OF ALTMAN-NYU SALOMON CENTER INDEXES OF DEFAULTED BONDS WITH OTHER SPECULATIVE SECURITIES INDEXES 1987 - 2002}

Correlation of Monthly Returns

\begin{tabular}{ccc}
$\begin{array}{c}\text { Altman-NYU } \\
\text { Bond Index }\end{array}$ & $\begin{array}{c}\text { S\&P 500 Stock } \\
\text { Index }\end{array}$ & $\begin{array}{c}\text { Salomon Smith } \\
\text { Barney High } \\
\text { Yield Index }\end{array}$ \\
\hline $100.00 \%$ & $27.59 \%$ & $60.16 \%$ \\
& $100.00 \%$ & $51.51 \%$ \\
& & $100.00 \%$ \\
\hline
\end{tabular}

Correlation of Quarterly Returns

Salomon Smith

Altman-NYU S\&P 500 Stock Barney High Bond Index Index Yield Index

Altman-NYU Bond Index

$100.00 \%$

$32.60 \%$

$58.10 \%$

S\&P 500 Stock Index

$100.00 \%$

$54.34 \%$

Salomon Smith Barney High Yield Index

$100.00 \%$ 


\section{FIGURE 13}

\section{CORRELATION OF ALTMAN-NYU SALOMON CENTER INDEXES OF DEFAULTED LOANS WITH OTHER SPECULATIVE \\ SECURITIES INDEXES 1996 - 2002}

\section{Correlation of Monthly Returns}

\begin{tabular}{cccc} 
& \multicolumn{3}{c}{ Salomon Smith } \\
Altman-NYU & S\&P 500 Stock & Barney High & Altman-NYU \\
Loan Index & Index & Yield Index & Bond Index
\end{tabular}

Altman-NYU Loan Index

$100.00 \%$

$-5.77 \%$

$41.77 \%$

$56.93 \%$

S\&P 500 Stock Index

$100.00 \%$

$51.54 \%$

$20.27 \%$

Salomon Smith Barney High Yield Index

$100.00 \%$

$61.13 \%$

Altman-NYU Bond Index

$100.00 \%$

\section{Correlation of Quarterly Returns}

Salomon Smith

Altman-NYU S\&P 500 Stock Barney High Altman-NYU

Loan Index Index Yield Index Bond Index

\begin{tabular}{|c|c|c|c|c|}
\hline Altman-NYU Loan Index & $100.00 \%$ & $9.14 \%$ & $40.18 \%$ & $58.32 \%$ \\
\hline S\&P 500 Stock Index & & $100.00 \%$ & $63.64 \%$ & $36.93 \%$ \\
\hline Salomon Smith Barney High Yield Index & & & $100.00 \%$ & $47.01 \%$ \\
\hline Altman-NYU Bond Index & & & & $100.00 \%$ \\
\hline
\end{tabular}

Source: Altman-NYU Salomon Center Defaulted Bond and Bank Loan Database 


\section{Figure 14}

Recovery Rate on Bank Loan Defaults: 1996 - 2002

(Prices at or Just After Default)

\begin{tabular}{rrrrrrr}
\hline & $\begin{array}{c}\text { Number of } \\
\text { New }\end{array}$ & $\begin{array}{c}\text { Number of } \\
\text { New }\end{array}$ & & Weighted & & $\begin{array}{c}\text { Standard } \\
\text { Deviation }\end{array}$ \\
Year & \multicolumn{1}{c}{ Issuers } & \multicolumn{1}{c}{$\begin{array}{c}\text { Facilities } \\
\text { Average }\end{array}$} & Median & Average & Devina \\
\hline 1996 & 7 & 9 & 86.00 & 80.42 & 73.34 & 24.41 \\
1997 & 4 & 4 & 87.88 & 94.86 & 87.92 & 12.61 \\
1998 & 4 & 5 & 72.00 & 84.70 & 75.77 & 18.34 \\
1999 & 20 & 40 & 51.25 & 54.44 & 56.31 & 22.96 \\
2000 & 18 & 41 & 65.00 & 59.36 & 66.06 & 16.69 \\
2001 & 39 & 95 & 64.50 & 57.40 & 59.51 & 20.69 \\
2002 & 37 & 68 & 67.16 & 51.98 & 65.65 & 21.66 \\
\hline & 129 & 262 & 70.54 & 69.02 & 69.22 & 19.62 \\
\hline \hline
\end{tabular}

Source: Altman-NYU Salomon Center Defaulted Bank Loan Database 


\section{Figure 15}

Recovery Rate on Bank Loan Defaults: 1999 - 2002

Telecommunications \& E-commerce only

(Prices at or Just After Default)

\begin{tabular}{|c|c|c|c|c|c|c|}
\hline Year & $\begin{array}{l}\text { Number of } \\
\text { New Issuers }\end{array}$ & $\begin{array}{c}\text { Number of } \\
\text { New } \\
\text { Facilities }\end{array}$ & Median & $\begin{array}{l}\text { Weighted } \\
\text { Average }\end{array}$ & Average & $\begin{array}{l}\text { Standard } \\
\text { Deviation }\end{array}$ \\
\hline 1999 & 2 & 4 & 46.33 & 45.69 & 46.17 & 0.84 \\
\hline 2000 & 2 & 7 & 51.00 & 60.62 & 64.21 & 19.49 \\
\hline 2001 & 13 & 34 & 67.00 & 55.00 & 59.54 & 24.65 \\
\hline 2002 & 8 & 19 & 69.00 & 65.07 & 58.93 & 21.66 \\
\hline & 25 & 64 & 58.33 & 56.60 & 57.21 & 16.66 \\
\hline
\end{tabular}

Source: Altman-NYU Salomon Center Defaulted Bank Loan Database 


\section{APPENDIX A}

\section{ALTMAN-NYU SALOMON CENTER INDEX OF DEFAULTED PUBLIC BONDS AND BANK LOANS}

Returns (1987 - 2000) and Comparison with

S\&P 500 stock index and Salomon Smith Barney High Yield Market Index

$($ December $1986=100)$

\begin{tabular}{|c|c|c|c|c|c|}
\hline MONTH & $\begin{array}{l}\text { PUBLIC } \\
\text { BOND } \\
\text { INDEX }\end{array}$ & $\begin{array}{c}\text { PUBLIC BOND } \\
\text { PERCENT } \\
\text { RETURN }\end{array}$ & $\begin{array}{l}\text { BANK LOAN } \\
\text { PERCENT } \\
\text { RETURN }\end{array}$ & $\begin{array}{c}\text { S\&P } \\
\text { PERCENT } \\
\text { RETURN }\end{array}$ & $\begin{array}{c}\text { SSMB-HYMI } \\
\text { PERCENT } \\
\text { RETURN }\end{array}$ \\
\hline JAN-87 & 109.80 & $9.80 \%$ & & $13.47 \%$ & $2.83 \%$ \\
\hline FEB-87 & 121.37 & $10.53 \%$ & & $3.95 \%$ & $1.65 \%$ \\
\hline MAR-87 & 125.95 & $3.77 \%$ & & $2.89 \%$ & $1.11 \%$ \\
\hline APR-87 & 127.52 & $1.25 \%$ & & $-0.89 \%$ & $-2.18 \%$ \\
\hline MAY-87 & 128.09 & $0.44 \%$ & & $0.87 \%$ & $-0.45 \%$ \\
\hline JUN-87 & 131.80 & $2.90 \%$ & & $5.05 \%$ & $1.38 \%$ \\
\hline JUL-87 & 139.05 & $5.50 \%$ & & $5.07 \%$ & $0.54 \%$ \\
\hline AUG-87 & 139.77 & $0.52 \%$ & & $3.73 \%$ & $1.00 \%$ \\
\hline SEP-87 & 136.35 & $-2.45 \%$ & & $-2.19 \%$ & $-2.30 \%$ \\
\hline OCT-87 & 124.19 & $-8.92 \%$ & & $-21.54 \%$ & $-2.67 \%$ \\
\hline NOV-87 & 128.19 & $3.22 \%$ & & $-8.24 \%$ & $2.53 \%$ \\
\hline DEC-87 & 137.85 & $7.53 \%$ & & $7.61 \%$ & $1.33 \%$ \\
\hline TOTAL 1987 RETURN & & $37.85 \%$ & & $5.26 \%$ & $4.67 \%$ \\
\hline JAN-88 & 139.84 & $1.44 \%$ & & $4.21 \%$ & $2.74 \%$ \\
\hline FEB-88 & 147.45 & $5.44 \%$ & & $4.66 \%$ & $2.71 \%$ \\
\hline MAR-88 & 152.01 & $3.10 \%$ & & $-3.09 \%$ & $-0.17 \%$ \\
\hline APR-88 & 156.85 & $3.18 \%$ & & $1.11 \%$ & $0.29 \%$ \\
\hline MAY-88 & 155.42 & $-0.91 \%$ & & $0.87 \%$ & $0.52 \%$ \\
\hline JUN-88 & 166.94 & $7.41 \%$ & & $4.59 \%$ & $1.91 \%$ \\
\hline JUL-88 & 165.05 & $-1.14 \%$ & & $-0.38 \%$ & $1.06 \%$ \\
\hline AUG-88 & 160.40 & $-2.82 \%$ & & $-3.40 \%$ & $0.33 \%$ \\
\hline SEP-88 & 160.28 & $-0.07 \%$ & & $4.26 \%$ & $1.01 \%$ \\
\hline OCT-88 & 157.69 & $-1.61 \%$ & & $2.78 \%$ & $1.56 \%$ \\
\hline NOV-88 & 166.88 & $5.83 \%$ & & $-1.43 \%$ & $0.38 \%$ \\
\hline DEC-88 & 174.36 & $4.48 \%$ & & $1.75 \%$ & $0.42 \%$ \\
\hline TOTAL 1988 RETURN & & $26.49 \%$ & & $16.61 \%$ & $13.47 \%$ \\
\hline JAN-89 & 166.57 & $-4.47 \%$ & & $7.32 \%$ & $1.75 \%$ \\
\hline FEB-89 & 159.93 & $-3.99 \%$ & & $-2.49 \%$ & $0.43 \%$ \\
\hline MAR-89 & 159.60 & $-0.21 \%$ & & $2.33 \%$ & $0.01 \%$ \\
\hline APR-89 & 162.88 & $2.06 \%$ & & $5.19 \%$ & $0.69 \%$ \\
\hline MAY-89 & 164.53 & $1.01 \%$ & & $4.05 \%$ & $1.70 \%$ \\
\hline JUN-89 & 164.38 & $-0.09 \%$ & & $-0.57 \%$ & $1.45 \%$ \\
\hline JUL-89 & 168.43 & $2.46 \%$ & & $9.03 \%$ & $0.45 \%$ \\
\hline AUG-89 & 164.96 & $-2.06 \%$ & & $1.96 \%$ & $-0.39 \%$ \\
\hline SEP-89 & 152.03 & $-7.84 \%$ & & $-0.41 \%$ & $-1.62 \%$ \\
\hline OCT-89 & 139.26 & $-8.40 \%$ & & $-2.32 \%$ & $-2.26 \%$ \\
\hline NOV-89 & 135.58 & $-2.64 \%$ & & $2.04 \%$ & $0.37 \%$ \\
\hline
\end{tabular}




\section{APPENDIX A}

\section{ALTMAN-NYU SALOMON CENTER INDEX OF DEFAULTED PUBLIC BONDS AND BANK LOANS}

Returns (1987 - 2000) and Comparison with

S\&P 500 stock index and Salomon Smith Barney High Yield Market Index

$($ December $1986=100)$

\begin{tabular}{|c|c|c|c|c|c|}
\hline MONTH & $\begin{array}{l}\text { PUBLIC } \\
\text { BOND } \\
\text { INDEX }\end{array}$ & $\begin{array}{c}\text { PUBLIC BOND } \\
\text { PERCENT } \\
\text { RETURN }\end{array}$ & $\begin{array}{l}\text { BANK LOAN } \\
\text { PERCENT } \\
\text { RETURN }\end{array}$ & $\begin{array}{c}\text { S\&P } \\
\text { PERCENT } \\
\text { RETURN }\end{array}$ & $\begin{array}{c}\text { SSMB-HYMI } \\
\text { PERCENT } \\
\text { RETURN }\end{array}$ \\
\hline DEC-89 & 134.64 & $-0.70 \%$ & & $2.40 \%$ & $0.22 \%$ \\
\hline TOTAL 1989 RETURN & & $-22.78 \%$ & & $31.68 \%$ & $2.75 \%$ \\
\hline JAN-90 & 130.72 & $-2.91 \%$ & & $-6.71 \%$ & $-3.03 \%$ \\
\hline FEB-90 & 127.03 & $-2.83 \%$ & & $1.29 \%$ & $-1.10 \%$ \\
\hline MAR-90 & 132.08 & $3.98 \%$ & & $2.65 \%$ & $1.06 \%$ \\
\hline APR-90 & 134.03 & $1.48 \%$ & & $-2.50 \%$ & $-0.51 \%$ \\
\hline MAY-90 & 132.37 & $-1.23 \%$ & & $9.75 \%$ & $2.63 \%$ \\
\hline JUN-90 & 130.12 & $-1.71 \%$ & & $-0.68 \%$ & $1.86 \%$ \\
\hline JUL-90 & 133.09 & $2.29 \%$ & & $-0.32 \%$ & $1.73 \%$ \\
\hline AUG-90 & 129.06 & $-3.03 \%$ & & $-9.04 \%$ & $-3.87 \%$ \\
\hline SEP-90 & 125.21 & $-2.99 \%$ & & $-4.87 \%$ & $-5.13 \%$ \\
\hline OCT-90 & 119.85 & $-4.28 \%$ & & $-0.43 \%$ & $-3.54 \%$ \\
\hline NOV-90 & 116.63 & $-2.69 \%$ & & $6.46 \%$ & $2.02 \%$ \\
\hline DEC-90 & 111.64 & $-4.27 \%$ & & $2.79 \%$ & $1.01 \%$ \\
\hline TOTAL 1990 RETURN & & $-17.08 \%$ & & $-3.12 \%$ & $-7.04 \%$ \\
\hline JAN-91 & 115.20 & $3.18 \%$ & & $4.36 \%$ & $2.59 \%$ \\
\hline FEB-91 & 124.97 & $8.49 \%$ & & $7.15 \%$ & $8.82 \%$ \\
\hline MAR-91 & 135.60 & $8.50 \%$ & & $2.42 \%$ & $5.24 \%$ \\
\hline APR-91 & 154.06 & $13.62 \%$ & & $0.24 \%$ & $3.75 \%$ \\
\hline MAY-91 & 158.67 & $2.99 \%$ & & $4.32 \%$ & $0.71 \%$ \\
\hline JUN-91 & 161.31 & $1.66 \%$ & & $-4.58 \%$ & $2.10 \%$ \\
\hline JUL-91 & 169.99 & $5.39 \%$ & & $4.66 \%$ & $2.85 \%$ \\
\hline AUG-91 & 167.79 & $-1.30 \%$ & & $2.37 \%$ & $2.33 \%$ \\
\hline SEP-91 & 165.36 & $-1.45 \%$ & & $-1.67 \%$ & $0.67 \%$ \\
\hline OCT-91 & 167.15 & $1.08 \%$ & & $1.34 \%$ & $3.00 \%$ \\
\hline NOV-91 & 165.61 & $-0.92 \%$ & & $-4.03 \%$ & $0.95 \%$ \\
\hline DEC-91 & 159.77 & $-3.53 \%$ & & $11.44 \%$ & $1.34 \%$ \\
\hline TOTAL 1991 RETURN & & $43.11 \%$ & & $30.48 \%$ & $39.93 \%$ \\
\hline JAN-92 & 171.04 & $7.06 \%$ & & $-1.86 \%$ & $2.89 \%$ \\
\hline FEB-92 & 176.52 & $3.21 \%$ & & $1.30 \%$ & $2.93 \%$ \\
\hline MAR-92 & 183.40 & $3.90 \%$ & & $-1.95 \%$ & $1.48 \%$ \\
\hline APR-92 & 182.90 & $-0.27 \%$ & & $2.94 \%$ & $0.74 \%$ \\
\hline MAY-92 & 187.59 & $2.57 \%$ & & $0.49 \%$ & $1.87 \%$ \\
\hline JUN-92 & 185.62 & $-1.05 \%$ & & $-1.49 \%$ & $1.24 \%$ \\
\hline JUL-92 & 186.09 & $0.25 \%$ & & $4.09 \%$ & $1.85 \%$ \\
\hline AUG-92 & 184.76 & $-0.72 \%$ & & $-2.05 \%$ & $1.33 \%$ \\
\hline SEP-92 & 183.03 & $-0.93 \%$ & & $1.18 \%$ & $0.93 \%$ \\
\hline
\end{tabular}




\section{APPENDIX A}

\section{ALTMAN-NYU SALOMON CENTER INDEX OF DEFAULTED PUBLIC BONDS AND BANK LOANS}

Returns (1987 - 2000) and Comparison with

S\&P 500 stock index and Salomon Smith Barney High Yield Market Index

$($ December $1986=100)$

\begin{tabular}{|c|c|c|c|c|c|}
\hline MONTH & $\begin{array}{l}\text { PUBLIC } \\
\text { BOND } \\
\text { INDEX }\end{array}$ & $\begin{array}{c}\text { PUBLIC BOND } \\
\text { PERCENT } \\
\text { RETURN }\end{array}$ & $\begin{array}{l}\text { BANK LOAN } \\
\text { PERCENT } \\
\text { RETURN } \\
\end{array}$ & $\begin{array}{c}\text { S\&P } \\
\text { PERCENT } \\
\text { RETURN } \\
\end{array}$ & $\begin{array}{c}\text { SSMB-HYMI } \\
\text { PERCENT } \\
\text { RETURN } \\
\end{array}$ \\
\hline OCT-92 & 181.53 & $-0.82 \%$ & & $0.35 \%$ & $-1.22 \%$ \\
\hline NOV-92 & 180.79 & $-0.41 \%$ & & $3.41 \%$ & $1.38 \%$ \\
\hline DEC-92 & 184.36 & $1.97 \%$ & & $1.23 \%$ & $1.19 \%$ \\
\hline TOTAL 1992 RETURN & & $15.39 \%$ & & $7.62 \%$ & $17.86 \%$ \\
\hline JAN-93 & 194.59 & $5.55 \%$ & & $0.84 \%$ & $2.44 \%$ \\
\hline FEB-93 & 200.59 & $3.09 \%$ & & $1.36 \%$ & $1.89 \%$ \\
\hline MAR-93 & 208.93 & $4.16 \%$ & & $2.11 \%$ & $1.55 \%$ \\
\hline APR-93 & 209.49 & $0.27 \%$ & & $-2.42 \%$ & $0.77 \%$ \\
\hline MAY-93 & 214.81 & $2.54 \%$ & & $2.68 \%$ & $1.38 \%$ \\
\hline JUN-93 & 218.68 & $1.80 \%$ & & $0.29 \%$ & $2.23 \%$ \\
\hline JUL-93 & 224.26 & $2.55 \%$ & & $-0.40 \%$ & $0.98 \%$ \\
\hline AUG-93 & 226.79 & $1.13 \%$ & & $3.79 \%$ & $1.08 \%$ \\
\hline SEP-93 & 229.73 & $1.30 \%$ & & $-0.77 \%$ & $0.24 \%$ \\
\hline OCT-93 & 231.21 & $0.64 \%$ & & $2.07 \%$ & $1.94 \%$ \\
\hline NOV-93 & 235.27 & $1.76 \%$ & & $-0.95 \%$ & $0.54 \%$ \\
\hline DEC-93 & 235.82 & $0.23 \%$ & & $1.21 \%$ & $1.10 \%$ \\
\hline TOTAL 1993 RETURN & & $27.91 \%$ & & $10.08 \%$ & $17.36 \%$ \\
\hline JAN-94 & 239.18 & $1.43 \%$ & & $3.40 \%$ & $2.17 \%$ \\
\hline FEB-94 & 246.84 & $3.20 \%$ & & $-2.71 \%$ & $-0.46 \%$ \\
\hline MAR-94 & 248.71 & $0.76 \%$ & & $-4.36 \%$ & $-3.72 \%$ \\
\hline APR-94 & 243.63 & $-2.04 \%$ & & $1.28 \%$ & $-0.91 \%$ \\
\hline MAY-94 & 246.53 & $1.19 \%$ & & $1.64 \%$ & $0.35 \%$ \\
\hline JUN-94 & 243.90 & $-1.06 \%$ & & $-2.45 \%$ & $0.11 \%$ \\
\hline JUL-94 & 245.06 & $0.47 \%$ & & $3.28 \%$ & $0.98 \%$ \\
\hline Aug-94 & 246.86 & $0.74 \%$ & & $4.10 \%$ & $0.56 \%$ \\
\hline Sep-94 & 250.31 & $1.40 \%$ & & $-2.45 \%$ & $-0.26 \%$ \\
\hline Oct-94 & 251.04 & $0.29 \%$ & & $2.25 \%$ & $0.02 \%$ \\
\hline Nov-94 & 252.28 & $0.50 \%$ & & $-3.64 \%$ & $-1.10 \%$ \\
\hline Dec-94 & 251.51 & $-0.30 \%$ & & $1.48 \%$ & $1.12 \%$ \\
\hline TOTAL 1994 RETURN & & $6.66 \%$ & & $1.32 \%$ & $-1.25 \%$ \\
\hline JAN-95 & 250.97 & $-0.22 \%$ & & $2.59 \%$ & $1.44 \%$ \\
\hline FEB-95 & 256.42 & $2.17 \%$ & & $3.90 \%$ & $3.33 \%$ \\
\hline MAR-95 & 267.27 & $4.23 \%$ & & $2.95 \%$ & $1.04 \%$ \\
\hline APR-95 & 267.51 & $0.09 \%$ & & $2.95 \%$ & $2.35 \%$ \\
\hline May-95 & 282.02 & $5.42 \%$ & & $4.00 \%$ & $2.98 \%$ \\
\hline June-95 & 281.51 & $-0.18 \%$ & & $2.32 \%$ & $0.71 \%$ \\
\hline July-95 & 282.02 & $0.18 \%$ & & $3.31 \%$ & $1.20 \%$ \\
\hline
\end{tabular}




\section{APPENDIX A}

\section{ALTMAN-NYU SALOMON CENTER INDEX OF DEFAULTED PUBLIC BONDS AND BANK LOANS}

Returns (1987 - 2000) and Comparison with

S\&P 500 stock index and Salomon Smith Barney High Yield Market Index

$($ December $1986=100)$

\begin{tabular}{|c|c|c|c|c|c|}
\hline MONTH & $\begin{array}{l}\text { PUBLIC } \\
\text { BOND } \\
\text { INDEX }\end{array}$ & $\begin{array}{c}\text { PUBLIC BOND } \\
\text { PERCENT } \\
\text { RETURN }\end{array}$ & $\begin{array}{l}\text { BANK LOAN } \\
\text { PERCENT } \\
\text { RETURN }\end{array}$ & $\begin{array}{c}\text { S\&P } \\
\text { PERCENT } \\
\text { RETURN }\end{array}$ & $\begin{array}{c}\text { SSMB-HYMI } \\
\text { PERCENT } \\
\text { RETURN }\end{array}$ \\
\hline Aug-95 & 282.10 & $0.03 \%$ & & $0.25 \%$ & $0.62 \%$ \\
\hline Sept-95 & 286.47 & $1.55 \%$ & & $4.22 \%$ & $1.16 \%$ \\
\hline Oct-95 & 273.01 & $-4.70 \%$ & & $-0.36 \%$ & $0.84 \%$ \\
\hline Nov-95 & 278.39 & $1.97 \%$ & & $4.39 \%$ & $0.91 \%$ \\
\hline Dec-95 & 279.84 & $0.52 \%$ & & $1.93 \%$ & $1.59 \%$ \\
\hline TOTAL 1995 RETURN & & $11.26 \%$ & & $37.56 \%$ & $19.71 \%$ \\
\hline Jan-96 & 286.86 & $2.51 \%$ & $0.96 \%$ & $3.40 \%$ & $1.47 \%$ \\
\hline Feb-96 & 309.09 & $7.75 \%$ & $2.80 \%$ & $0.93 \%$ & $0.62 \%$ \\
\hline Mar-96 & 323.11 & $4.54 \%$ & $2.79 \%$ & $0.96 \%$ & $-0.50 \%$ \\
\hline Apr-96 & 329.51 & $1.98 \%$ & $-0.01 \%$ & $1.47 \%$ & $-0.03 \%$ \\
\hline May-96 & 333.71 & $1.28 \%$ & $4.87 \%$ & $2.58 \%$ & $0.56 \%$ \\
\hline Jun-96 & 344.77 & $3.31 \%$ & $3.76 \%$ & $0.38 \%$ & $0.77 \%$ \\
\hline Jul-96 & 340.99 & $-1.09 \%$ & $1.38 \%$ & $-4.42 \%$ & $0.65 \%$ \\
\hline Aug-96 & 341.81 & $0.24 \%$ & $-1.14 \%$ & $2.11 \%$ & $1.04 \%$ \\
\hline Sep-96 & 349.01 & $2.11 \%$ & $0.79 \%$ & $5.63 \%$ & $2.34 \%$ \\
\hline Oct-96 & 355.63 & $1.90 \%$ & $1.69 \%$ & $2.76 \%$ & $1.15 \%$ \\
\hline Nov-96 & 324.98 & $-8.62 \%$ & $0.37 \%$ & $7.56 \%$ & $1.92 \%$ \\
\hline Dec-96 & 308.40 & $-5.10 \%$ & $-0.10 \%$ & $-1.98 \%$ & $0.79 \%$ \\
\hline TOTAL 1996 RETURN & & $10.21 \%$ & $19.56 \%$ & $22.96 \%$ & $11.29 \%$ \\
\hline Jan-97 & 303.64 & $-1.54 \%$ & $1.88 \%$ & $6.25 \%$ & $0.75 \%$ \\
\hline Feb-97 & 308.02 & $1.44 \%$ & $2.40 \%$ & $0.78 \%$ & $1.70 \%$ \\
\hline Mar-97 & 313.37 & $1.74 \%$ & $0.77 \%$ & $-4.11 \%$ & $-1.03 \%$ \\
\hline Apr-97 & 306.69 & $-2.13 \%$ & $-6.63 \%$ & $5.97 \%$ & $0.71 \%$ \\
\hline May-97 & 307.03 & $0.11 \%$ & $-1.93 \%$ & $6.88 \%$ & $2.02 \%$ \\
\hline Jun-97 & 305.28 & $-0.57 \%$ & $3.60 \%$ & $4.48 \%$ & $1.69 \%$ \\
\hline Jul-97 & 304.59 & $-0.23 \%$ & $0.45 \%$ & $7.96 \%$ & $2.29 \%$ \\
\hline Aug-97 & 311.49 & $2.27 \%$ & $1.19 \%$ & $-5.60 \%$ & $0.25 \%$ \\
\hline Sep-97 & 316.60 & $1.64 \%$ & $2.41 \%$ & $5.48 \%$ & $1.75 \%$ \\
\hline Oct-97 & 315.20 & $-0.44 \%$ & $0.24 \%$ & $-3.34 \%$ & $0.80 \%$ \\
\hline Nov-97 & 311.57 & $-1.15 \%$ & $-0.41 \%$ & $4.63 \%$ & $0.51 \%$ \\
\hline Dec-97 & 303.53 & $-2.58 \%$ & $-1.82 \%$ & $1.72 \%$ & $1.05 \%$ \\
\hline TOTAL 1997 RETURN & & $-1.58 \%$ & $1.75 \%$ & $34.36 \%$ & $13.18 \%$ \\
\hline Jan-98 & 303.5 & $0.00 \%$ & $-0.38 \%$ & $1.11 \%$ & $2.26 \%$ \\
\hline Feb-98 & 309.5 & $1.96 \%$ & $-0.84 \%$ & $7.21 \%$ & $0.68 \%$ \\
\hline Mar-98 & 312.0 & $0.82 \%$ & $1.68 \%$ & $5.12 \%$ & $1.08 \%$ \\
\hline Apr-98 & 312.6 & $0.19 \%$ & $4.19 \%$ & $1.01 \%$ & $0.54 \%$ \\
\hline May-98 & 319.7 & $2.27 \%$ & $2.33 \%$ & $-1.72 \%$ & $0.27 \%$ \\
\hline
\end{tabular}




\section{APPENDIX A}

\section{ALTMAN-NYU SALOMON CENTER INDEX OF DEFAULTED PUBLIC BONDS AND BANK LOANS}

Returns (1987 - 2000) and Comparison with

S\&P 500 stock index and Salomon Smith Barney High Yield Market Index

$($ December $1986=100)$

\begin{tabular}{|c|c|c|c|c|c|}
\hline MONTH & $\begin{array}{l}\text { PUBLIC } \\
\text { BOND } \\
\text { INDEX }\end{array}$ & $\begin{array}{c}\text { PUBLIC BOND } \\
\text { PERCENT } \\
\text { RETURN }\end{array}$ & $\begin{array}{l}\text { BANK LOAN } \\
\text { PERCENT } \\
\text { RETURN }\end{array}$ & $\begin{array}{c}\text { S\&P } \\
\text { PERCENT } \\
\text { RETURN }\end{array}$ & $\begin{array}{c}\text { SSMB-HYMI } \\
\text { PERCENT } \\
\text { RETURN }\end{array}$ \\
\hline Jun-98 & 318.8 & $-0.28 \%$ & $-0.99 \%$ & $4.06 \%$ & $0.22 \%$ \\
\hline Jul-98 & 322.5 & $1.15 \%$ & $-0.05 \%$ & $-1.07 \%$ & $0.80 \%$ \\
\hline Aug-98 & 263.6 & $-18.25 \%$ & $-6.26 \%$ & $-14.46 \%$ & $-6.70 \%$ \\
\hline Sep-98 & 234.1 & $-11.21 \%$ & $-6.16 \%$ & $6.41 \%$ & $1.23 \%$ \\
\hline Oct-98 & 211.9 & $-9.48 \%$ & $-7.88 \%$ & $8.13 \%$ & $-1.38 \%$ \\
\hline Nov-98 & 227.4 & $7.32 \%$ & $5.44 \%$ & $6.06 \%$ & $5.02 \%$ \\
\hline Dec-98 & 221.9 & $-2.43 \%$ & $-0.85 \%$ & $5.76 \%$ & $-0.07 \%$ \\
\hline 1998 YTD & & $-26.91 \%$ & $-10.22 \%$ & $28.58 \%$ & $3.60 \%$ \\
\hline Jan-99 & 222.3 & $0.22 \%$ & $3.59 \%$ & $4.18 \%$ & $1.50 \%$ \\
\hline Feb-99 & 231.0 & $3.91 \%$ & $-1.01 \%$ & $-3.11 \%$ & $-0.84 \%$ \\
\hline Mar-99 & 242.8 & $5.07 \%$ & $-1.70 \%$ & $4.00 \%$ & $0.85 \%$ \\
\hline Apr-99 & 269.8 & $11.15 \%$ & $2.91 \%$ & $3.87 \%$ & $2.09 \%$ \\
\hline May-99 & 266.7 & $-1.14 \%$ & $1.92 \%$ & $-2.36 \%$ & $-1.57 \%$ \\
\hline Jun-99 & 269.4 & $1.00 \%$ & $2.58 \%$ & $5.50 \%$ & $-0.22 \%$ \\
\hline Jul-99 & 279.5 & $3.75 \%$ & $1.31 \%$ & $-3.12 \%$ & $0.22 \%$ \\
\hline Aug-99 & 265.6 & $-4.96 \%$ & $-4.80 \%$ & $-0.50 \%$ & $-1.19 \%$ \\
\hline Sep-99 & 251.5 & $-5.33 \%$ & $1.29 \%$ & $-2.74 \%$ & $-0.76 \%$ \\
\hline Oct-99 & 233.5 & $-7.13 \%$ & $-2.64 \%$ & $6.33 \%$ & $-0.68 \%$ \\
\hline Nov-99 & 249.3 & $6.75 \%$ & $-2.31 \%$ & $2.04 \%$ & $1.57 \%$ \\
\hline Dec-99 & 247.0 & $-0.92 \%$ & $-0.12 \%$ & $5.89 \%$ & $0.84 \%$ \\
\hline 1999 YTD & & $11.34 \%$ & $0.65 \%$ & $20.98 \%$ & $1.74 \%$ \\
\hline Jan-00 & 255.7 & $3.50 \%$ & $3.64 \%$ & $-5.02 \%$ & $-0.83 \%$ \\
\hline Feb-00 & 253.1 & $-1.01 \%$ & $-2.27 \%$ & $-1.89 \%$ & $0.24 \%$ \\
\hline Mar-00 & 245.9 & $-2.86 \%$ & $-5.48 \%$ & $9.77 \%$ & $-2.03 \%$ \\
\hline Apr-00 & 232.0 & $-5.64 \%$ & $1.02 \%$ & $-3.01 \%$ & $0.40 \%$ \\
\hline Мay-00 & 219.3 & $-5.46 \%$ & $-0.08 \%$ & $-2.05 \%$ & $-1.39 \%$ \\
\hline Jun-00 & 221.9 & $1.16 \%$ & $-1.46 \%$ & $2.47 \%$ & $2.25 \%$ \\
\hline Jul-00 & 221.2 & $-0.32 \%$ & $0.38 \%$ & $-1.56 \%$ & $1.09 \%$ \\
\hline Aug-00 & 212.4 & $-3.96 \%$ & $-0.65 \%$ & $6.21 \%$ & $0.74 \%$ \\
\hline Sep-00 & 211.1 & $-0.64 \%$ & $0.86 \%$ & $-5.28 \%$ & $-1.07 \%$ \\
\hline Oct-00 & 196.5 & $-6.91 \%$ & $-0.71 \%$ & $-0.42 \%$ & $-2.96 \%$ \\
\hline Nov-00 & 176.6 & $-10.10 \%$ & $-1.39 \%$ & $-7.88 \%$ & $-4.09 \%$ \\
\hline Dec-00 & 165.3 & $-6.42 \%$ & $-0.39 \%$ & $0.49 \%$ & $2.04 \%$ \\
\hline 2000 YTD & & $-33.09 \%$ & $-6.59 \%$ & $-9.11 \%$ & $-5.68 \%$ \\
\hline Jan-01 & 172.8 & $4.55 \%$ & $4.11 \%$ & $3.55 \%$ & $6.74 \%$ \\
\hline Feb-01 & 183.0 & $5.90 \%$ & $6.71 \%$ & $-9.11 \%$ & $1.19 \%$ \\
\hline Mar-01 & 173.1 & $-5.43 \%$ & $0.57 \%$ & $-6.33 \%$ & $-1.82 \%$ \\
\hline
\end{tabular}




\section{APPENDIX A}

\section{ALTMAN-NYU SALOMON CENTER INDEX OF DEFAULTED PUBLIC BONDS AND BANK LOANS}

Returns (1987 - 2000) and Comparison with

S\&P 500 stock index and Salomon Smith Barney High Yield Market Index

$($ December $1986=100)$

\begin{tabular}{cccccc} 
& $\begin{array}{c}\text { PUBLIC } \\
\text { BOND }\end{array}$ & $\begin{array}{c}\text { PUBLIC BOND } \\
\text { PERCENT }\end{array}$ & $\begin{array}{c}\text { BANK LOAN } \\
\text { PERCENT }\end{array}$ & $\begin{array}{c}\text { S\&P } \\
\text { PERCENT }\end{array}$ & $\begin{array}{c}\text { SSMB-HYMI } \\
\text { PERENT } \\
\text { REETURN }\end{array}$ \\
\hline MONTH & 168.6 & $-2.57 \%$ & $-2.31 \%$ & $7.76 \%$ & $-1.47 \%$ \\
Apr-01 & 180.7 & $7.20 \%$ & $2.04 \%$ & $0.67 \%$ & $1.61 \%$ \\
May-01 & 189.7 & $4.96 \%$ & $1.27 \%$ & $-2.43 \%$ & $-2.75 \%$ \\
Jun-01 & 191.9 & $1.17 \%$ & $-0.22 \%$ & $-0.98 \%$ & $1.88 \%$ \\
Jul-01 & 195.8 & $2.04 \%$ & $3.05 \%$ & $-6.25 \%$ & $1.37 \%$ \\
Aug-01 & 180.5 & $-7.84 \%$ & $-1.99 \%$ & $-8.07 \%$ & $-7.20 \%$ \\
Sep-01 & 190.2 & $5.35 \%$ & $-1.73 \%$ & $1.91 \%$ & $3.32 \%$ \\
Oct-01 & 193.1 & $1.57 \%$ & $1.12 \%$ & $7.67 \%$ & $3.80 \%$ \\
Nov-01 & 194.1 & $0.52 \%$ & $0.89 \%$ & $0.88 \%$ & $-0.64 \%$ \\
Dec-01 & & $17.47 \%$ & $13.94 \%$ & $-11.87 \%$ & $5.44 \%$ \\
\hline 2001 YTD & 197.8 & $1.90 \%$ & $3.51 \%$ & $-1.46 \%$ & $0.43 \%$ \\
\hline Jan-02 & 189.4 & $-4.26 \%$ & $-1.43 \%$ & $-1.93 \%$ & $-1.09 \%$ \\
Feb-02 & 193.4 & $2.12 \%$ & $3.71 \%$ & $3.76 \%$ & $2.66 \%$ \\
Mar-02 & 195.9 & $1.29 \%$ & $3.27 \%$ & $-6.06 \%$ & $1.64 \%$ \\
Apr-02 & 191.7 & $-2.17 \%$ & $0.21 \%$ & $-0.73 \%$ & $-1.24 \%$ \\
May-02 & 173.6 & $-9.44 \%$ & $-1.64 \%$ & $-7.12 \%$ & $-8.81 \%$ \\
Jun-02 & 166.3 & $-4.19 \%$ & $-4.27 \%$ & $-7.79 \%$ & $-4.52 \%$ \\
Jul-02 & 176.4 & $6.05 \%$ & $-4.37 \%$ & $0.66 \%$ & $3.81 \%$ \\
Aug-02 & 174.4 & $-1.11 \%$ & $-2.03 \%$ & $-10.86 \%$ & $-1.97 \%$ \\
Sep-02 & 172.2 & $-1.27 \%$ & $-1.25 \%$ & $8.80 \%$ & $-0.34 \%$ \\
Oct-02 & 186.8 & $8.49 \%$ & $5.37 \%$ & $5.88 \%$ & $7.63 \%$ \\
Nov-02 & 182.5 & $-2.28 \%$ & $2.51 \%$ & $-5.87 \%$ & $1.21 \%$ \\
Dec-02 & $-5.98 \%$ & $3.03 \%$ & $-22.08 \%$ & $-1.53 \%$ \\
\hline 2002 YTD & & & & RETURN $\%$ \\
\hline
\end{tabular}

Source: Altman-NYU Salomon Center Defaulted Bond and Bank Loan Databases 


\section{APPENDIX B}

\section{ALTMAN-NYU SALOMON CENTER INDEX OF DEFAULTED BANK LOANS}

Returns (1996 - 2002) and Comparison with

S\&P 500 Stock Index and Salomon Smith Barney High Yield Market Index

** Base of 100 starting December 1995 for Altman Index**

\begin{tabular}{|c|c|c|c|c|c|}
\hline MONTH & $\begin{array}{l}\text { BANK } \\
\text { LOAN } \\
\text { INDEX }\end{array}$ & $\begin{array}{l}\text { BANK LOAN } \\
\text { PERCENT } \\
\text { RETURN }\end{array}$ & $\begin{array}{c}\text { PUBLIC BOND } \\
\text { PERCENT } \\
\text { RETURN }\end{array}$ & $\begin{array}{c}\text { S\&P } \\
\text { PERCENT } \\
\text { RETURN }\end{array}$ & $\begin{array}{c}\text { SSMB } \\
\text { PERCENT } \\
\text { RETURN }\end{array}$ \\
\hline Jan-96 & 101.0 & $0.96 \%$ & $2.51 \%$ & $3.40 \%$ & $1.58 \%$ \\
\hline Feb-96 & 103.8 & $2.80 \%$ & $7.75 \%$ & $0.93 \%$ & $0.15 \%$ \\
\hline Mar-96 & 106.7 & $2.79 \%$ & $4.54 \%$ & $0.96 \%$ & $-0.27 \%$ \\
\hline Apr-96 & 106.7 & $-0.01 \%$ & $1.98 \%$ & $1.47 \%$ & $0.05 \%$ \\
\hline May-96 & 111.9 & $4.87 \%$ & $1.28 \%$ & $2.58 \%$ & $0.72 \%$ \\
\hline Jun-96 & 116.1 & $3.76 \%$ & $3.31 \%$ & $0.38 \%$ & $0.60 \%$ \\
\hline Jul-96 & 117.7 & $1.38 \%$ & $-1.09 \%$ & $-4.42 \%$ & $0.68 \%$ \\
\hline Aug-96 & 116.3 & $-1.14 \%$ & $0.24 \%$ & $2.11 \%$ & $1.03 \%$ \\
\hline Sep-96 & 117.3 & $0.79 \%$ & $2.11 \%$ & $5.63 \%$ & $2.15 \%$ \\
\hline Oct-96 & 119.2 & $1.69 \%$ & $1.90 \%$ & $2.76 \%$ & $1.10 \%$ \\
\hline Nov-96 & 119.7 & $0.37 \%$ & $-8.62 \%$ & $7.56 \%$ & $2.02 \%$ \\
\hline Dec-96 & 119.6 & $-0.10 \%$ & $-5.10 \%$ & $-1.98 \%$ & $0.77 \%$ \\
\hline TOTAL 1996 RETURN & & $19.56 \%$ & $10.21 \%$ & $22.96 \%$ & $11.06 \%$ \\
\hline Jan-97 & 121.8 & $1.88 \%$ & $-1.54 \%$ & $6.25 \%$ & $0.52 \%$ \\
\hline Feb-97 & 124.7 & $2.40 \%$ & $1.44 \%$ & $0.78 \%$ & $1.43 \%$ \\
\hline Mar-97 & 125.7 & $0.77 \%$ & $1.74 \%$ & $-4.11 \%$ & $-1.11 \%$ \\
\hline Apr-97 & 117.4 & $-6.63 \%$ & $-2.13 \%$ & $5.97 \%$ & $1.14 \%$ \\
\hline May-97 & 115.1 & $-1.93 \%$ & $0.11 \%$ & $6.88 \%$ & $2.15 \%$ \\
\hline Jun-97 & 119.2 & $3.60 \%$ & $-0.57 \%$ & $4.48 \%$ & $1.52 \%$ \\
\hline Jul-97 & 119.8 & $0.45 \%$ & $-0.23 \%$ & $7.96 \%$ & $2.40 \%$ \\
\hline Aug-97 & 121.2 & $1.19 \%$ & $2.27 \%$ & $-5.60 \%$ & $-0.18 \%$ \\
\hline Sep-97 & 124.1 & $2.41 \%$ & $1.64 \%$ & $5.48 \%$ & $1.66 \%$ \\
\hline Oct-97 & 124.4 & $0.24 \%$ & $-0.44 \%$ & $-3.34 \%$ & $0.66 \%$ \\
\hline Nov-97 & 123.9 & $-0.41 \%$ & $-1.15 \%$ & $4.63 \%$ & $0.95 \%$ \\
\hline Dec-97 & 121.6 & $-1.82 \%$ & $-2.58 \%$ & $1.72 \%$ & $0.95 \%$ \\
\hline TOTAL 1997 RETURN & & $1.75 \%$ & $-1.58 \%$ & $34.36 \%$ & $12.73 \%$ \\
\hline Jan-98 & 121.2 & $-0.38 \%$ & $0.00 \%$ & $1.11 \%$ & $2.26 \%$ \\
\hline Feb-98 & 120.2 & $-0.84 \%$ & $1.96 \%$ & $7.21 \%$ & $0.68 \%$ \\
\hline Mar-98 & 122.2 & $1.68 \%$ & $0.82 \%$ & $5.12 \%$ & $1.08 \%$ \\
\hline Apr-98 & 127.3 & $4.19 \%$ & $0.19 \%$ & $1.01 \%$ & $0.54 \%$ \\
\hline May-98 & 130.3 & $2.33 \%$ & $2.27 \%$ & $-1.72 \%$ & $0.27 \%$ \\
\hline Jun-98 & 129.0 & $-0.99 \%$ & $-0.28 \%$ & $4.06 \%$ & $0.22 \%$ \\
\hline Jul-98 & 128.9 & $-0.05 \%$ & $1.15 \%$ & $-1.07 \%$ & $0.80 \%$ \\
\hline
\end{tabular}




\section{APPENDIX B}

\section{ALTMAN-NYU SALOMON CENTER INDEX OF DEFAULTED BANK LOANS}

Returns (1996 - 2002) and Comparison with

S\&P 500 Stock Index and Salomon Smith Barney High Yield Market Index

** Base of 100 starting December 1995 for Altman Index**

\begin{tabular}{|c|c|c|c|c|c|}
\hline MONTH & $\begin{array}{l}\text { BANK } \\
\text { LOAN } \\
\text { INDEX }\end{array}$ & $\begin{array}{c}\text { BANK LOAN } \\
\text { PERCENT } \\
\text { RETURN }\end{array}$ & $\begin{array}{c}\text { PUBLIC BOND } \\
\text { PERCENT } \\
\text { RETURN }\end{array}$ & $\begin{array}{c}\text { S\&P } \\
\text { PERCENT } \\
\text { RETURN }\end{array}$ & $\begin{array}{c}\text { SSMB } \\
\text { PERCENT } \\
\text { RETURN } \\
\end{array}$ \\
\hline Aug-98 & 120.8 & $-6.26 \%$ & $-18.25 \%$ & $-14.46 \%$ & $-6.70 \%$ \\
\hline Sep-98 & 113.4 & $-6.16 \%$ & $-11.21 \%$ & $6.41 \%$ & $1.23 \%$ \\
\hline Oct-98 & 104.5 & $-7.88 \%$ & $-9.48 \%$ & $8.13 \%$ & $-1.38 \%$ \\
\hline Nov-98 & 110.2 & $5.44 \%$ & $7.32 \%$ & $6.06 \%$ & $5.02 \%$ \\
\hline Dec-98 & 109.2 & $-0.85 \%$ & $-2.43 \%$ & $5.76 \%$ & $-0.07 \%$ \\
\hline 1998 YTD & & $-10.22 \%$ & $-26.91 \%$ & $28.58 \%$ & $3.60 \%$ \\
\hline Jan-99 & 113.1 & $3.59 \%$ & $0.22 \%$ & $4.18 \%$ & $1.50 \%$ \\
\hline Feb-99 & 112.0 & $-1.01 \%$ & $3.91 \%$ & $-3.11 \%$ & $-0.84 \%$ \\
\hline Mar-99 & 110.1 & $-1.70 \%$ & $5.07 \%$ & $4.00 \%$ & $0.85 \%$ \\
\hline Apr-99 & 113.3 & $2.91 \%$ & $11.15 \%$ & $3.87 \%$ & $2.09 \%$ \\
\hline May-99 & 115.5 & $1.92 \%$ & $-1.14 \%$ & $-2.36 \%$ & $-1.57 \%$ \\
\hline Jun-99 & 118.5 & $2.58 \%$ & $1.00 \%$ & $5.50 \%$ & $-0.22 \%$ \\
\hline Jul-99 & 120.0 & $1.31 \%$ & $3.75 \%$ & $-3.12 \%$ & $0.22 \%$ \\
\hline Aug-99 & 114.2 & $-4.80 \%$ & $-4.96 \%$ & $-0.50 \%$ & $-1.19 \%$ \\
\hline Sep-99 & 115.7 & $1.29 \%$ & $-5.33 \%$ & $-2.74 \%$ & $-0.76 \%$ \\
\hline Oct-99 & 112.7 & $-2.64 \%$ & $-7.13 \%$ & $6.33 \%$ & $-0.68 \%$ \\
\hline Nov-99 & 110.1 & $-2.31 \%$ & $6.75 \%$ & $2.04 \%$ & $1.57 \%$ \\
\hline Dec-99 & 109.9 & $-0.12 \%$ & $-0.92 \%$ & $5.89 \%$ & $0.84 \%$ \\
\hline 1999 YTD & & $0.65 \%$ & $11.34 \%$ & $20.98 \%$ & $1.74 \%$ \\
\hline Jan-00 & 113.9 & $3.64 \%$ & $3.50 \%$ & $-5.02 \%$ & $-0.83 \%$ \\
\hline Feb-00 & 111.3 & $-2.27 \%$ & $-1.01 \%$ & $-1.89 \%$ & $0.24 \%$ \\
\hline Mar-00 & 105.2 & $-5.48 \%$ & $-2.86 \%$ & $9.77 \%$ & $-2.03 \%$ \\
\hline Apr-00 & 106.3 & $1.02 \%$ & $-5.64 \%$ & $-3.01 \%$ & $0.40 \%$ \\
\hline May-00 & 106.2 & $-0.08 \%$ & $-5.46 \%$ & $-2.05 \%$ & $-1.39 \%$ \\
\hline Jun-00 & 104.7 & $-1.46 \%$ & $1.16 \%$ & $2.47 \%$ & $2.25 \%$ \\
\hline Jul-00 & 105.1 & $0.38 \%$ & $-0.32 \%$ & $-1.56 \%$ & $1.09 \%$ \\
\hline Aug-00 & 104.4 & $-0.65 \%$ & $-3.96 \%$ & $6.21 \%$ & $0.74 \%$ \\
\hline Sep-00 & 105.3 & $0.86 \%$ & $-0.64 \%$ & $-5.28 \%$ & $-1.07 \%$ \\
\hline Oct-00 & 104.5 & $-0.71 \%$ & $-6.91 \%$ & $-0.42 \%$ & $-2.96 \%$ \\
\hline Nov-00 & 103.1 & $-1.39 \%$ & $-10.10 \%$ & $-7.88 \%$ & $-4.09 \%$ \\
\hline Dec-00 & 102.7 & $-0.39 \%$ & $-6.42 \%$ & $0.49 \%$ & $2.04 \%$ \\
\hline 2000 YTD & & $-6.59 \%$ & $-33.09 \%$ & $-9.11 \%$ & $-5.68 \%$ \\
\hline Jan-01 & 106.9 & $4.11 \%$ & $4.55 \%$ & $3.55 \%$ & $6.74 \%$ \\
\hline Feb-01 & 114.1 & $6.71 \%$ & $5.90 \%$ & $-9.11 \%$ & $1.19 \%$ \\
\hline
\end{tabular}




\section{APPENDIX B}

\section{ALTMAN-NYU SALOMON CENTER INDEX OF DEFAULTED BANK LOANS}

Returns (1996 - 2002) and Comparison with

S\&P 500 Stock Index and Salomon Smith Barney High Yield Market Index

** Base of 100 starting December 1995 for Altman Index**

\begin{tabular}{|c|c|c|c|c|c|}
\hline MONTH & $\begin{array}{l}\text { BANK } \\
\text { LOAN } \\
\text { INDEX }\end{array}$ & $\begin{array}{c}\text { BANK LOAN } \\
\text { PERCENT } \\
\text { RETURN }\end{array}$ & $\begin{array}{c}\text { PUBLIC BOND } \\
\text { PERCENT } \\
\text { RETURN }\end{array}$ & $\begin{array}{c}\text { S\&P } \\
\text { PERCENT } \\
\text { RETURN }\end{array}$ & $\begin{array}{c}\text { SSMB } \\
\text { PERCENT } \\
\text { RETURN }\end{array}$ \\
\hline Mar-01 & 114.7 & $0.57 \%$ & $-5.43 \%$ & $-6.33 \%$ & $-1.82 \%$ \\
\hline Apr-01 & 112.1 & $-2.31 \%$ & $-2.57 \%$ & $7.76 \%$ & $-1.47 \%$ \\
\hline May-01 & 114.3 & $2.04 \%$ & $7.20 \%$ & $0.67 \%$ & $1.61 \%$ \\
\hline Jun-01 & 115.8 & $1.27 \%$ & $4.96 \%$ & $-2.43 \%$ & $-2.75 \%$ \\
\hline Jul-01 & 115.5 & $-0.22 \%$ & $1.17 \%$ & $-0.98 \%$ & $1.88 \%$ \\
\hline Aug-01 & 119.1 & $3.05 \%$ & $2.04 \%$ & $-6.25 \%$ & $1.37 \%$ \\
\hline Sep-01 & 116.7 & $-1.99 \%$ & $-7.84 \%$ & $-8.07 \%$ & $-7.20 \%$ \\
\hline Oct-01 & 114.7 & $-1.73 \%$ & $5.35 \%$ & $1.91 \%$ & $3.32 \%$ \\
\hline Nov-01 & 116.0 & $1.12 \%$ & $1.57 \%$ & $7.67 \%$ & $3.80 \%$ \\
\hline Dec-01 & 117.0 & $0.89 \%$ & $0.52 \%$ & $0.88 \%$ & $-0.64 \%$ \\
\hline 2001 YTD & & $13.94 \%$ & $17.47 \%$ & $-11.87 \%$ & $5.44 \%$ \\
\hline Jan-02 & 121.1 & $3.51 \%$ & $1.90 \%$ & $-1.46 \%$ & $0.43 \%$ \\
\hline Feb-02 & 119.4 & $-1.43 \%$ & $-4.26 \%$ & $-1.93 \%$ & $-1.09 \%$ \\
\hline Mar-02 & 123.8 & $3.71 \%$ & $2.12 \%$ & $3.76 \%$ & $2.66 \%$ \\
\hline Apr-02 & 127.8 & $3.27 \%$ & $1.29 \%$ & $-6.06 \%$ & $1.64 \%$ \\
\hline May-02 & 128.1 & $0.21 \%$ & $-2.17 \%$ & $-0.73 \%$ & $-1.24 \%$ \\
\hline Jun-02 & 126.0 & $-1.64 \%$ & $-9.44 \%$ & $-7.12 \%$ & $-8.81 \%$ \\
\hline Jul-02 & 120.6 & $-4.27 \%$ & $-4.19 \%$ & $-7.79 \%$ & $-4.52 \%$ \\
\hline Aug-02 & 115.3 & $-4.37 \%$ & $6.05 \%$ & $0.66 \%$ & $3.81 \%$ \\
\hline Sep-02 & 113.0 & $-2.03 \%$ & $-1.11 \%$ & $-10.86 \%$ & $-1.97 \%$ \\
\hline Oct-02 & 111.6 & $-1.25 \%$ & $-1.27 \%$ & $8.80 \%$ & $-0.34 \%$ \\
\hline Nov-02 & 117.6 & $5.37 \%$ & $8.49 \%$ & $5.88 \%$ & $7.63 \%$ \\
\hline Dec-02 & 120.5 & $2.51 \%$ & $-2.28 \%$ & $-5.87 \%$ & $1.21 \%$ \\
\hline 2002 YTD & & $3.03 \%$ & $-5.98 \%$ & $-22.08 \%$ & $-1.53 \%$ \\
\hline
\end{tabular}

Source: Altman-NYU Salomon Center Defaulted Bond and Bank Loan Databases 
APPENDIX C

Combined Altman-NYU Salomon Center

Defaulted Public Bond And Bank Loan Index

$($ December $1995=100)$

\begin{tabular}{|c|c|c|c|}
\hline \multirow[b]{2}{*}{ Date } & \multicolumn{2}{|c|}{ Monthly } & \multirow{2}{*}{$\begin{array}{c}\text { Year-to-Date } \\
\text { Return }\end{array}$} \\
\hline & Level & Return & \\
\hline Dec-95 & 100.0 & & \\
\hline Jan-96 & 101.8 & $1.80 \%$ & $1.80 \%$ \\
\hline Feb-96 & 107.1 & $5.21 \%$ & $7.09 \%$ \\
\hline Mar-96 & 111.1 & $3.71 \%$ & $11.05 \%$ \\
\hline Apr-96 & 112.2 & $1.04 \%$ & $12.23 \%$ \\
\hline May-96 & 115.7 & $3.13 \%$ & $15.74 \%$ \\
\hline Jun-96 & 119.8 & $3.53 \%$ & $19.82 \%$ \\
\hline Jul-96 & 119.8 & $0.02 \%$ & $19.84 \%$ \\
\hline Aug-96 & 119.0 & $-0.67 \%$ & $19.04 \%$ \\
\hline Sep-96 & 120.8 & $1.47 \%$ & $20.79 \%$ \\
\hline Oct-96 & 123.0 & $1.80 \%$ & $22.97 \%$ \\
\hline Nov-96 & 118.3 & $-3.80 \%$ & $18.29 \%$ \\
\hline Dec-96 & 115.6 & $-2.26 \%$ & $15.62 \%$ \\
\hline Jan-97 & 116.2 & $0.48 \%$ & $0.48 \%$ \\
\hline Feb-97 & 118.6 & $2.04 \%$ & $2.54 \%$ \\
\hline Mar-97 & 119.9 & $1.15 \%$ & $3.71 \%$ \\
\hline Apr-97 & 114.6 & $-4.39 \%$ & $-0.83 \%$ \\
\hline Мay-97 & 113.7 & $-0.85 \%$ & $-1.68 \%$ \\
\hline Jun-97 & 115.4 & $1.51 \%$ & $-0.19 \%$ \\
\hline Jul-97 & 115.6 & $0.19 \%$ & $-0.08 \%$ \\
\hline Aug-97 & 117.5 & $1.72 \%$ & $1.64 \%$ \\
\hline Sep-97 & 119.8 & $2.01 \%$ & $3.67 \%$ \\
\hline Oct-97 & 119.7 & $-0.13 \%$ & $3.54 \%$ \\
\hline Nov-97 & 118.7 & $-0.79 \%$ & $2.72 \%$ \\
\hline Dec-97 & 116.1 & $-2.22 \%$ & $0.44 \%$ \\
\hline Jan-98 & 115.9 & $-0.20 \%$ & $-0.20 \%$ \\
\hline Feb-98 & 116.3 & $0.38 \%$ & $0.18 \%$ \\
\hline Mar-98 & 117.8 & $1.32 \%$ & $1.50 \%$ \\
\hline Apr-98 & 121.0 & $2.67 \%$ & $4.21 \%$ \\
\hline May-98 & 123.8 & $2.31 \%$ & $6.61 \%$ \\
\hline Jun-98 & 123.0 & $-0.66 \%$ & $5.91 \%$ \\
\hline Jul-98 & 123.6 & $0.51 \%$ & $6.45 \%$ \\
\hline Aug-98 & 109.2 & $-11.67 \%$ & $-5.97 \%$ \\
\hline Sep-98 & 100.0 & $-8.37 \%$ & $-13.83 \%$ \\
\hline Oct-98 & 91.5 & $-8.52 \%$ & $-21.18 \%$ \\
\hline Nov-98 & 97.2 & $6.20 \%$ & $-16.29 \%$ \\
\hline Dec-98 & 95.7 & $-1.51 \%$ & $-17.55 \%$ \\
\hline Jan-99 & 97.6 & $1.97 \%$ & $1.97 \%$ \\
\hline Feb-99 & 98.4 & $0.78 \%$ & $2.77 \%$ \\
\hline Mar-99 & 99.2 & $0.84 \%$ & $3.63 \%$ \\
\hline Apr-99 & 105.5 & $6.31 \%$ & $10.16 \%$ \\
\hline
\end{tabular}


APPENDIX C

Combined Altman-NYU Salomon Center

Defaulted Public Bond And Bank Loan Index

$($ December $1995=100)$

\begin{tabular}{|c|c|c|c|}
\hline \multirow[b]{2}{*}{ Date } & \multicolumn{2}{|c|}{ Monthly } & \multirow{2}{*}{$\begin{array}{c}\text { Year-to-Date } \\
\text { Return }\end{array}$} \\
\hline & Level & Return & \\
\hline May-99 & 105.9 & $0.45 \%$ & $10.66 \%$ \\
\hline Jun-99 & 107.7 & $1.66 \%$ & $12.49 \%$ \\
\hline Jul-99 & 110.4 & $2.49 \%$ & $15.29 \%$ \\
\hline Aug-99 & 105.0 & $-4.87 \%$ & $9.67 \%$ \\
\hline Sep-99 & 103.3 & $-1.58 \%$ & $7.94 \%$ \\
\hline Oct-99 & 98.8 & $-4.34 \%$ & $3.26 \%$ \\
\hline Nov-99 & 100.4 & $1.59 \%$ & $4.90 \%$ \\
\hline Dec-99 & 100.0 & $-0.43 \%$ & $4.45 \%$ \\
\hline Jan-00 & 103.6 & $3.59 \%$ & $3.59 \%$ \\
\hline Feb-00 & 101.7 & $-1.78 \%$ & $1.74 \%$ \\
\hline Mar-00 & 97.3 & $-4.31 \%$ & $-2.64 \%$ \\
\hline Apr-00 & 95.6 & $-1.80 \%$ & $-4.39 \%$ \\
\hline May-00 & 93.4 & $-2.26 \%$ & $-6.55 \%$ \\
\hline Jun-00 & 93.1 & $-0.41 \%$ & $-6.93 \%$ \\
\hline Jul-00 & 93.1 & $0.10 \%$ & $-6.85 \%$ \\
\hline Aug-00 & 91.3 & $-2.02 \%$ & $-8.73 \%$ \\
\hline Sep-00 & 91.4 & $0.13 \%$ & $-8.61 \%$ \\
\hline Oct-00 & 89.1 & $-2.51 \%$ & $-10.90 \%$ \\
\hline Nov-00 & 85.7 & $-3.77 \%$ & $-14.26 \%$ \\
\hline Dec-00 & 84.2 & $-1.84 \%$ & $-15.84 \%$ \\
\hline Jan-01 & 87.7 & $4.21 \%$ & $4.21 \%$ \\
\hline Feb-01 & 93.4 & $6.50 \%$ & $10.99 \%$ \\
\hline Mar-01 & 92.3 & $-1.19 \%$ & $9.67 \%$ \\
\hline Apr-01 & 90.1 & $-2.39 \%$ & $7.05 \%$ \\
\hline May-01 & 93.4 & $3.65 \%$ & $10.96 \%$ \\
\hline Jun-01 & 95.6 & $2.42 \%$ & $13.64 \%$ \\
\hline Jul-01 & 95.8 & $0.18 \%$ & $13.84 \%$ \\
\hline Aug-01 & 98.4 & $2.73 \%$ & $16.95 \%$ \\
\hline Sep-01 & 94.9 & $-3.61 \%$ & $12.73 \%$ \\
\hline Oct-01 & 95.3 & $0.45 \%$ & $13.24 \%$ \\
\hline Nov-01 & 96.5 & $1.27 \%$ & $14.68 \%$ \\
\hline Dec-01 & 97.2 & $0.77 \%$ & $15.56 \%$ \\
\hline Jan-02 & 100.1 & $2.96 \%$ & $2.96 \%$ \\
\hline Feb-02 & 97.7 & $-2.38 \%$ & $0.52 \%$ \\
\hline Mar-02 & 100.9 & $3.18 \%$ & $3.71 \%$ \\
\hline Apr-02 & 103.5 & $2.60 \%$ & $6.41 \%$ \\
\hline May-02 & 102.8 & $-0.68 \%$ & $5.68 \%$ \\
\hline Jun-02 & 97.6 & $-5.10 \%$ & $0.32 \%$ \\
\hline Jul-02 & 93.4 & $-4.24 \%$ & $-3.93 \%$ \\
\hline Aug-02 & 92.9 & $-0.54 \%$ & $-4.45 \%$ \\
\hline Sep-02 & 91.3 & $-1.70 \%$ & $-6.07 \%$ \\
\hline
\end{tabular}


APPENDIX C

Combined Altman-NYU Salomon Center

Defaulted Public Bond And Bank Loan Index

$($ December $1995=100)$

\begin{tabular}{|crcc|}
\hline \multirow{2}{*}{ Date } & \multicolumn{2}{c}{ Monthly } & Year-to-Date \\
Oct-02 & 90.2 & $-1.26 \%$ & $-7.25 \%$ \\
Nov-02 & 96.1 & $6.51 \%$ & $-1.22 \%$ \\
Dec-02 & 96.7 & $0.69 \%$ & $-0.53 \%$ \\
\hline
\end{tabular}

Source: Altman-NYU Salomon Center Defaulted Bond and Bank Loan Databases 
Angelo, Gordon \& Company

Apollo Management

Appaloosa Management

Ashmore Asian Recovery

Avenue Capital Partners

Bay Harbour Advisors

Bennett Management Company

Black Diamond

Blackstone Alternative Asset Management

Canyon Capital

Cardinal Capital

Cargill

Carl Marks

Cerebrus Partners

Citadel Investments

Concordia Advisors

Contrarian Capital

Davidson Kempner

DDJ Capital Management

Epic Asset Management
Farallon Partners

Franklin Mutual Series

Golden Tree

GSC Capital

Halcyon/Slika (Alan B.) Management

HBV/Mellon

King Street Advisors

KPS Special Situations Fund

KS Distressed Debt

Lampe Conway

Leucadia National Corporation

LongAcre Capital Partners

Levco Debt Opportunities

MatlinPatterson Global Advisors

MD Sass Corporate Resurgence

MHR

MJ Whitman Mgmt Co.

Moore Asian Recovery Fund

Murray Capital

Oaktree Capital
Pacholder Associates, Inc.

Pacific Alternative Asset Management

Pequot Capital

PPM America

Resolution Partners

Restoration Capital Management

Satellite

Schultze Asset Management

Silverpoint Capital

Stanfield Capital Management

Strategic Value Partners

TA Mckay \& Co.

Trust Company of the West

Turnberry Capital

Varde Partners, Inc.

Wellspring Capital Partners

Wexford Capital

Weyland Fund

Whippoorwill Associates, Inc.

William E. Simon \& Sons

W.R. Ross \& Co. 


\section{APPENDIX E}

Investment Styles in Distressed Debt Investing

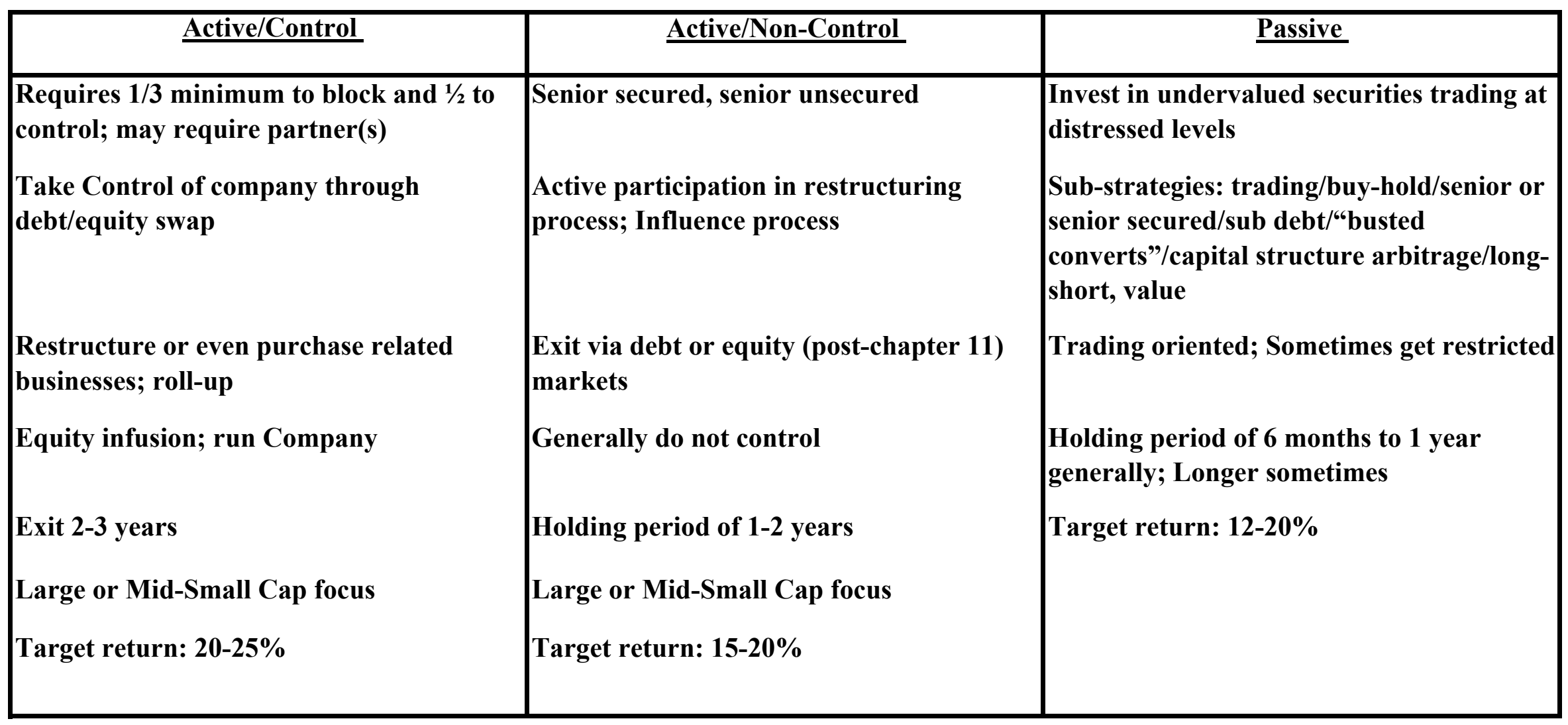

